\title{
TWO NEW BURSTING NEUTRON STAR LOW-MASS X-RAY BINARIES: SWIFT J185003.2-005627 AND SWIFT J1922.7-1716
}

\author{
N. Degenaar ${ }^{1,4}$, M. Linares ${ }^{2}$, D. Altamirano ${ }^{3}$, And R. Wijnands $^{3}$ \\ ${ }^{1}$ Department of Astronomy, University of Michigan, 500 Church St, Ann Arbor, MI 48109, USA; degenaar@umich.edu \\ ${ }^{2}$ Massachusetts Institute of Technology (MIT), Kavli Institute for Astrophysics and Space Research, Cambridge, MA 02139, USA \\ ${ }^{3}$ Astronomical Institute "Anton Pannekoek," University of Amsterdam, Postbus 94249, 1090 GE Amsterdam, The Netherlands \\ Received 2012 July 18; accepted 2012 September 5; published 2012 October 11
}

\begin{abstract}
We discuss the origin of two triggers of Swift's Burst Alert Telescope (BAT) that occurred in 2011. The triggers were identified with Swift J185003.2-005627, a previously unknown X-ray source, and the known but unclassified X-ray transient Swift J1922.7-1716. We investigate the BAT data and follow-up observations obtained with Swift's $\mathrm{X}$-ray and ultraviolet/optical telescopes to demonstrate that both triggers are consistent with thermonuclear X-ray bursts. This implies that both sources are neutron star low-mass $X$-ray binaries. The total duration of $\simeq 7$ minutes and estimated energy output of $\simeq(3-7) \times 10^{39}$ erg fall in between that of normal and intermediately long X-ray bursts. From the observed peaks of the X-ray bursts, we estimate a distance of $\lesssim 3.7 \mathrm{kpc}$ for Swift J185003.2-005627 and $\lesssim 4.8 \mathrm{kpc}$ for Swift J1922.7-1716. We characterize the outburst and quiescent X-ray properties of the two sources. They have comparable average outburst luminosities of $\simeq 10^{35-36} \mathrm{erg} \mathrm{s}^{-1}$, and a quiescent luminosity equal to or lower than $\simeq 2 \times 10^{32} \mathrm{erg} \mathrm{s}^{-1}(0.5-10 \mathrm{keV})$. Swift J185003.2-005627 returned to quiescence $\simeq 20 \mathrm{~d}$ after its BAT trigger, while Swift J1922.7-1716 appears to exhibit long accretion outbursts that last several months to years. We identify a unique counterpart for Swift J1922.7-1716 in the ultraviolet/optical data. Finally, we serendipitously detect a flare lasting $\simeq 500 \mathrm{~s}$ from an uncataloged X-ray/optical object that we tentatively classify as a flaring M-dwarf.
\end{abstract}

Key words: accretion, accretion disks - stars: flare - stars: neutron - X-rays: binaries - X-rays: individual (Swift J1922.7-1716, Swift J185003.2-005627)

\section{INTRODUCTION}

Low-mass X-ray binaries (LMXBs) consist of a neutron star or a black hole that accretes matter from a (sub-)solar mass companion star that overflows its Roche lobe. The accretion process involves the formation of an accretion disk and typically generates a $0.5-10 \mathrm{keV}$ X-ray luminosity of $L_{X} \simeq 10^{36-39} \mathrm{erg} \mathrm{s}^{-1}$ (e.g., Chen et al. 1997), which places LMXBs amongst the brightest X-ray point sources in our Galaxy. Many LMXBs have an unstable accretion disk (see Lasota 2001, for a review). This causes the system to become transient, i.e., to alternate accretion outbursts with long episodes of quiescence during which the $\mathrm{X}$-ray emission is much dimmer $\left(\lesssim 10^{33} \mathrm{erg} \mathrm{s}^{-1}\right.$; e.g., Menou et al. 1999; Garcia et al. 2001). Typically, the active phases of transient LMXBs last for a few weeks or months, while they reside in quiescence for years before a new outburst commences.

Whereas it is not straightforward to distinguish the nature of the compact object from the X-ray spectral and timing properties of the outburst (e.g., van der Klis 1994; Linares et al. 2007), there are two phenomena that require a solid surface and are therefore considered a distinctive property of accreting neutron stars. These are coherent X-ray pulsations and thermonuclear $\mathrm{X}$-ray bursts (i.e., type-I X-ray bursts, X-ray bursts hereafter). $\mathrm{X}$-ray bursts are bright flashes of X-ray emission that may reach up to the Eddington limit and temporarily outshine the accretion luminosity. They are caused by unstable burning of accreted helium $(\mathrm{He})$ and/or hydrogen $(\mathrm{H})$ on the surface of the neutron star.

$\mathrm{X}$-ray bursts are characterized by blackbody emission that peaks at a temperature up to $k T_{\mathrm{bb}} \simeq 2-3 \mathrm{keV}$ and decays to $\simeq 1 \mathrm{keV}$ as the $\mathrm{X}$-ray burst fades. The observable properties such

\footnotetext{
4 Hubble fellow.
}

as the duration $\left(t_{b}\right)$, radiated energy $\left(E_{b}\right)$, and recurrence time $\left(t_{\mathrm{rec}}\right)$ depend on the amount and composition of the fuel that is consumed during the $\mathrm{X}$-ray burst. The most commonly observed $\mathrm{X}$-ray bursts last for $t_{b} \simeq 10-100 \mathrm{~s}$, have a radiated energy output of $E_{b} \simeq 10^{39} \mathrm{erg}$, and recur on a timescale of minutes to days. To date, thousands of such events have been observed from about $\simeq 100$ different LMXBs (see, e.g., Cornelisse et al. 2003; Galloway et al. 2008).

Apart from these normal X-ray bursts, there are two classes of more energetic events that are also more rare: intermediately long X-ray bursts $\left(t_{b} \simeq\right.$ tens of minutes to hours, $E_{b} \simeq$ $10^{40-41} \mathrm{erg}$, and $t_{\mathrm{rec}} \simeq$ weeks to years; see Chenevez et al. 2008; Falanga et al. 2008) and superbursts ( $t_{b} \simeq$ hours to days, $E_{b} \simeq 10^{42} \mathrm{erg}$, and $t_{\text {rec }} \simeq$ a year; see Kuulkers 2004; Keek \& in 't Zand 2008). To date, only a few tens of such energetic X-ray bursts have been observed from about two dozens of LMXBs.

In this work, we discuss the properties of Swift J185003.2-005627 and Swift J1922.7-1716. We demonstrate that both sources displayed an X-ray burst in 2011 that triggered Swift's Burst Alert Telescope (BAT; Barthelmy et al. 2005). We use rapid follow-up observations obtained with the narrow-field X-Ray Telescope (XRT; Burrows et al. 2005) and UltraViolet/Optical Telescope (UVOT; Roming et al. 2005) to fully characterize the trigger events. In addition, we investigate the outburst and the quiescent properties of both sources.

\subsection{The New X-Ray Source Swift J185003.2-005627}

On 2011 June 24, Swift/BAT triggered and located an unknown source (trigger 456014; Beardmore et al. 2011a). Based on the soft nature of the BAT detection and the proximity to the Galactic plane, the trigger was tentatively identified as a previously unknown Galactic transient and dubbed Swift 
J185003.2-005627 (J1850 hereafter). The detection of a fading X-ray counterpart in follow-up XRT observations provided an arcsecond localization of the source (Beardmore et al. 2011a). Based on the properties of the XRT data and the refined analysis of the BAT trigger data, ${ }^{5}$ it was suggested that this event was a thermonuclear X-ray burst (Markwardt et al. 2011; Beardmore et al. 2011b).

J1850 was not detected in the Swift/BAT hard X-ray transient monitor on or after the time of the BAT trigger, with a $1 \sigma$ upper limit on the daily averaged $15-50 \mathrm{keV}$ count rate of $7 \times 10^{-4}$ counts $\mathrm{s}^{-1} \mathrm{~cm}^{-2}$ (Krimm et al. 2011). However, the source was detected at an average $15-50 \mathrm{keV}$ intensity of $(3.5 \pm 1.0) \times 10^{-3}$ counts $\mathrm{s}^{-1} \mathrm{~cm}^{-2}$ (corresponding to a flux of $\simeq 2 \times 10^{-10} \mathrm{erg} \mathrm{cm}^{-2} \mathrm{~s}^{-1}$ for a Crab-like spectrum) between 2011 May 18 and May 26. Archival searches back to 2005 February did not reveal any similar detections of J1850 (Krimm et al. 2011).

\subsection{The Unclassified Source Swift J1922.7-1716}

Swift J1922.7-1716 (hereafter J1922) is a transient X-ray source that was discovered during the Swift/BAT hard X-ray survey of 2004 December-2005 March (Tueller et al. 2005a, 2005b). Swift/XRT follow-up observations allowed for the identification of a soft X-ray counterpart (Tueller et al. 2005b). Non-detections with INTEGRAL (2003-2004; Falanga et al. 2006) and Swift (2006 October-November; Kennea et al. 2011) testified to its transient nature.

Falanga et al. (2006) discussed Swift, RXTE, and INTEGRAL data taken between 2005 July and November. The broadband spectrum and timing properties were found to be typical of an LMXB in a faint X-ray state, but the nature of the compact accretor (i.e., a neutron star or a black hole) could not be established. A combined blackbody and power-law model provided an adequate description of the broadband spectrum, with a hydrogen column density $N_{\mathrm{H}} \simeq(1.5-2.0) \times 10^{21} \mathrm{~cm}^{-2}$, spectral index $\Gamma \simeq 1.4-1.8$, and temperature $k T_{\mathrm{bb}} \simeq 0.4-0.6 \mathrm{keV}$. The resulting $0.1-100 \mathrm{keV}$ flux was $\simeq(3.3-3.9) \times 10^{-10} \mathrm{erg} \mathrm{cm}^{-2} \mathrm{~s}^{-1}$ (Falanga et al. 2006).

Renewed activity of $\mathrm{J} 1922$ was seen with MAXI and Swift/XRT in 2011 August (Nakahira et al. 2011; Kennea et al. 2011). Inspection of the Swift/BAT transient monitor data revealed that the source was detected in hard X-rays starting in 2011 July. It was seen at a mean $15-50 \mathrm{keV}$ count rate of $(1.5 \pm 0.3) \times 10^{-3}$ counts s${ }^{-1} \mathrm{~cm}^{-2}\left(\simeq 1 \times 10^{-10} \mathrm{erg} \mathrm{cm}^{-2} \mathrm{~s}^{-1}\right.$ for a Crab-like spectrum) until early-August, after which the hard X-ray flux decreased (Kennea et al. 2011). The 2011 intensity was similar to the average BAT count rate of the source in 2005.

Swift/BAT triggered on J1922 on 2011 November 3 (Barthelmy et al. 2011). ${ }^{6}$ Preliminary analysis of the BAT and XRT data revealed that the trigger was very likely caused by an X-ray burst (Degenaar et al. 2011d). Optical spectroscopy revealed clear $\mathrm{He}$ - and $\mathrm{H}$-emission lines, supporting an LMXB nature (Wiersema et al. 2011; Halpern \& Skinner 2011). The presence of H-lines implies that the donor star is H-rich and therefore the binary orbital period must be $P_{\text {orb }} \gtrsim 1.5 \mathrm{hr}$ (Nelson et al. 1986). Upper limits on the quiescent optical counterpart ( $>23.4$ mag in the $r$ and $g$ bands) are consistent with an M-dwarf or evolved star and suggest $P_{\text {orb }} \lesssim 5 \mathrm{hr}$ (Halpern \& Skinner 2011).

\footnotetext{
5 See also http://gcn.gsfc.nasa.gov/notices_s/456014/BA/

6 See also http://gcn.gsfc.nasa.gov/notices_s/506913/BA/
}

\section{OBSERVATIONS AND DATA ANALYSIS}

We use publicly available Swift data to investigate the BAT triggers and outburst properties of J1850 and J1922. In addition, we analyze archival XMM-Newton and Suzaku data in an attempt to constrain their quiescent emission level. Table 1 gives an overview of all observations discussed in this work. The observations, data reduction, and analysis procedures are detailed in the following sections. All errors quoted in this work refer to $90 \%$ confidence levels unless stated otherwise.

\subsection{Swift/BAT}

We generated standard BAT data products $(15-150 \mathrm{keV})$ for the trigger observations using the BATGRBPRODUCT tool. In all cases, the spacecraft started slewing toward the trigger location when the source had already faded into the background. We extracted single BAT spectra of the pre-slew data employing the tool BATBINEVT. The standard geometrical corrections and the BAT-recommended systematical error were applied with BATUPDATEPHAKW and BATPHASYSERR, respectively. Since we use only pre-slew data, we generated a single response matrix for each trigger by running the task BATDRMGEN. The spectra were fitted between 15 and $35 \mathrm{keV}$ with XSPEC (ver. 12.7; Arnaud 1996).

\subsection{Swift/XRT}

The XRT data cover an energy range of $0.5-10 \mathrm{keV}$ and consist of a combination of photon counting (PC) and windowed timing (WT) mode. In the PC mode, a two-dimensional image is acquired, whereas in the WT mode, the CCD columns are collapsed into a one-dimensional image to reduce the frame time. The WT is typically used when the count rate exceeds $\simeq 1-2$ counts $\mathrm{s}^{-1}$, because higher rates cause considerable pileup in the PC mode.

All Swift data were reduced using the Swift tools (ver. 3.8) within the HEASOFT package (ver. 6.12), and employing the latest calibration data (ver. 3.8). We made use of the online tools to obtain XRT data products and obtain a global characterization of the persistent emission (Evans et al. 2009). ${ }^{7}$ For the detailed analysis of the X-ray bursts, we manually extracted XRT light curves and spectra using XSELECT (ver. 3.8). Exposure maps were generated using the tool XRTEXPOMAP, and subsequently used to create ancillary response files (arfs) with XRTMAKEARF. The latest redistribution matrix files (rmfs) were taken from the calibration database (CALDB).

The XRT spectra were grouped to contain a minimum of 20 photons per bin and fitted between 0.5 and $10 \mathrm{keV}$ in XSPEC.

\subsection{Swift/UVOT}

The UVOT data were obtained using a variety of optical and ultraviolet (UV) filters in a wavelength range of $\simeq 1500-8500 \AA$ (see Poole et al. 2008). We used a standard aperture of $5^{\prime \prime}$ to extract source photons and a source-free region with a radius of $10^{\prime \prime}$ as a background reference. Magnitudes and light curves were extracted using the tools UVOTSOURCE and UVOTMAGHIST.

\subsection{Spectral Analysis and Eddington Limit}

For our spectral analysis with XSPEC, we use a blackbody model (BBODYRAD), a simple power law (POWERLAW), or a combination of both. In all fits, we included the PHABS model to

\footnotetext{
http://www.swift.ac.uk/user_objects/
} 
Table 1

Log of (Soft) X-Ray Observations

\begin{tabular}{llll}
\hline \hline Satellite/Instrument & Obs ID & Date & $\begin{array}{r}t_{\mathrm{exp}} \\
(\mathrm{ks})\end{array}$
\end{tabular}

(ks)

\begin{tabular}{|c|c|c|c|}
\hline \multicolumn{4}{|c|}{ Swift J185003.2-005627 } \\
\hline XMM-Newton/EPIC* & 73740101 & 2003 Mar 21 & 26.8 \\
\hline XMM-Newton/EPIC* & 73740201 & 2003 Mar 23 & 20.5 \\
\hline XMM-Newton/EPIC* & 73740301 & 2003 Apr 18 & 24.2 \\
\hline Swift/XRT (PC+WT) & 456014000 & 2011 Jun 24 & 1.8 \\
\hline Swift/XRT (PC+WT) & 456014001 & 2011 Jun 25 & 1.0 \\
\hline Swift/XRT (PC+WT) & 456014002 & 2011 Jun 25 & 1.5 \\
\hline Swift/XRT (PC+WT) & 456014003 & 2011 Jun 25 & 1.5 \\
\hline Swift/XRT (WT) & 456014004 & 2011 Jun 26 & 1.0 \\
\hline Swift/XRT (WT) & 456014005 & 2011 Jun 27 & 1.5 \\
\hline Swift/XRT (WT) & 456014007 & 2011 Jun 28 & 4.9 \\
\hline Swift/XRT (WT) & 456014008 & 2011 Jun 29 & 1.0 \\
\hline Swift/XRT (WT) & 456014010 & 2011 Jul 1 & 1.0 \\
\hline Swift/XRT (WT) & 456014011 & 2011 Jul 2 & 0.4 \\
\hline Swift/XRT (WT) & 456014012 & 2011 Jul 3 & 1.2 \\
\hline Swift/XRT (WT) & 456014013 & $2011 \mathrm{Jul} 4$ & 1.0 \\
\hline Swift/XRT (WT) & 456014014 & 2011 Jul 5 & 0.5 \\
\hline Swift/XRT (WT) & 456014015 & $2011 \mathrm{Jul} 6$ & 1.0 \\
\hline Swift/XRT (WT) & 456014016 & 2011 Jul 7 & 1.0 \\
\hline Swift/XRT (WT) & 456014017 & 2011 Jul 8 & 0.9 \\
\hline Swift/XRT (WT) & 456014019 & 2011 Jul 10 & 0.5 \\
\hline Swift/XRT (PC) & 456014021 & 2011 Jul 12 & 1.1 \\
\hline Swift/XRT (PC) & 456014022 & 2011 Jul 13 & 1.0 \\
\hline Swift/XRT (PC)* & 456014023 & 2011 Jul 16 & 0.2 \\
\hline Swift/XRT (PC)* & 456014024 & 2011 Jul 17 & 0.8 \\
\hline Swift/XRT (PC)* & 456014025 & 2011 Jul 18 & 0.8 \\
\hline Swift/XRT (PC)* & 456014026 & 2011 Jul 19 & 0.4 \\
\hline Swift/XRT (PC)* & 456014027 & 2011 Jul 20 & 0.1 \\
\hline Swift/XRT (PC)* & 456014028 & 2011 Jul 21 & 1.0 \\
\hline \multicolumn{4}{|c|}{ Swift J1922.7-1716 } \\
\hline Swift/XRT $(\mathrm{PC}+\mathrm{WT}) \dagger$ & 35174001 & 2005 Jul 8 & 6.4 \\
\hline Swift/XRT $(\mathrm{PC}+\mathrm{WT}) \dagger$ & 35174003 & 2005 Oct 1 & 10.8 \\
\hline Swift/XRT (PC+WT) & 35471001 & 2006 Mar 14 & 16.2 \\
\hline Swift/XRT (PC+WT) & 35471002 & 2006 Jun 4 & 0.3 \\
\hline Swift/XRT (PC+WT) & 35471003 & 2006 Jun 18 & 10.8 \\
\hline Swift/XRT (PC)* & 35471004 & 2006 Oct 30 & 5.2 \\
\hline Swift/XRT (PC)* & 35471005 & 2006 Nov 3 & 1.1 \\
\hline Swift/XRT (PC)* & 35471006 & 2006 Nov 10 & 4.4 \\
\hline Swift/XRT (PC)* & 35471007 & 2006 Nov 14 & 3.7 \\
\hline Suzaku/XIS* & 702028010 & 2007 Apr 10 & 78.6 \\
\hline Swift/XRT (PC+WT) & 35471008 & 2011 Aug 13 & 1.0 \\
\hline Swift/XRT (PC+WT) & 35471009 & 2011 Aug 30 & 1.2 \\
\hline Swift/XRT (PC+WT) & 35471010 & 2011 Aug 31 & 4.2 \\
\hline Swift/XRT (WT) & 506913000 & 2011 Nov 3 & 1.9 \\
\hline Swift/XRT (WT) & 35471011 & 2011 Nov 4 & 0.9 \\
\hline Swift/XRT (WT) & 35471012 & 2011 Nov 5 & 1.0 \\
\hline Swift/XRT (PC+WT) & 35471013 & 2012 Mar 9 & 0.7 \\
\hline Swift/XRT (PC+WT) & 35471014 & 2012 Mar 14 & 1.0 \\
\hline Swift/XRT (PC)* & 35471015 & 2012 May 25 & 1.2 \\
\hline Swift/XRT (PC)* & 35471016 & 2012 Jun 9 & 1.9 \\
\hline Swift/XRT (PC)* & 35471017 & 2012 Jun 11 & 2.0 \\
\hline Swift/XRT (PC)* & 35471018 & 2012 Jun 13 & 0.9 \\
\hline Swift/XRT (PC)* & 35471019 & 2012 Jun 15 & 0.7 \\
\hline Swift/XRT (PC)* & 35471020 & 2012 Jun 17 & 0.3 \\
\hline Swift/XRT (PC)* & 35471021 & 2012 Jun 21 & 2.3 \\
\hline
\end{tabular}

Notes. Observations marked by an asterisk were obtained during quiescent episodes. The two observations marked by a dagger were also discussed by Falanga et al. (2006).

account for neutral hydrogen absorption along the line of sight, for which we employed the default XSPEC abundances (Anders
\& Grevesse 1989) and cross-sections (Balucinska-Church \& McCammon 1992).

The bolometric accretion luminosity is typically a factor $\simeq 2-3$ higher than observed in the $0.5-10 \mathrm{keV}$ energy band (in 't Zand et al. 2007). We therefore apply a correction factor of 2.5 to the $0.5-10 \mathrm{keV}$ luminosity ( $L_{X}$, determined from the Swift/XRT data) to estimate the bolometric accretion luminosity $\left(L_{\text {bol }}\right)$. We subsequently use this to estimate the mass-accretion rate onto the neutron star (during outburst) via the relation $\dot{M}_{\mathrm{ob}}=R L_{\mathrm{bol}} / G M$, where $G=6.67 \times 10^{-8} \mathrm{~cm}^{3} \mathrm{~g}^{-1} \mathrm{~s}^{-2}$ is the gravitational constant, and $M$ and $R$ are the mass and radius of the neutron star, respectively. We adopt canonical values of $M=1.4 M_{\odot}$ and $R=10 \mathrm{~km}$.

It is often useful to express the accretion luminosity and mass-accretion rate in terms of the Eddington limit. We will adopt $L_{\mathrm{EDD}}=3.8 \times 10^{38} \mathrm{erg} \mathrm{s}^{-1}$, which is the empirical limit determined from $\mathrm{X}$-ray bursts that display photospheric radius expansion (PRE; Kuulkers et al. 2003).

\section{RESULTS FOR SWIFT J185003.2-005627}

\subsection{Outburst Properties}

\subsubsection{XRT}

Figure 1 shows the outburst evolution of J1850 as seen with Swift/XRT. Following the BAT trigger on 2011 June 24, the source was observed almost daily for 27 days until 2011 July 21 . In the days following the X-ray burst, the intensity remained steady at $\simeq 5$ counts $\mathrm{s}^{-1}$ (WT), but the source started fading around 2011 July 5 (11 days after the BAT trigger). It eventually went undetected with the XRT starting on 2011 July 16 (22 days after the BAT trigger). Considering the sensitivity of the XRT, a non-detection in $\simeq 3.3 \mathrm{ks}$ of data between 2011 July 16 and 21 (Table 1) suggests that the source luminosity had dropped below a few times $10^{33} \mathrm{erg} \mathrm{s}^{-1}$ (depending on the spectral shape, $0.5-10 \mathrm{keV})$. This indicates that the outburst had ceased and that the source had returned to quiescence. We further constrain the quiescent emission in Section 3.3.

The outburst observed with the XRT had a duration of $\simeq 3$ weeks, but the BAT already detected activity from the source on 2011 May 18-26 (see Section 1). If this was part of the same outburst, then it had a duration of $\gtrsim 8$ weeks. Lack of X-ray data before this epoch does not allow us to constrain the onset of the activity any further.

To obtain a global characterization of the outburst spectrum and flux, we fitted the PC and WT data simultaneously. The hydrogen column density was fixed between the two data sets, whereas the other fit parameters were left to vary freely. The results of our spectral analysis are summarized in Table 2.

Fitting the spectra with either a single power-law or blackbody model yields reduced chi-square values of $\chi_{v}^{2} \gtrsim 1.3$ for 673 degrees of freedom (dof). A combined power-law and blackbody model provides a better description of the data and results in $N_{\mathrm{H}} \simeq(1.1 \pm 0.1) \times 10^{22} \mathrm{~cm}^{-2}, \Gamma \simeq 0.9-1.6$, and $k T_{\mathrm{bb}} \simeq 0.7 \mathrm{keV}\left(\chi_{v}^{2}=1.1\right.$ for $669 \mathrm{dof}$; Table 2$)$. The blackbody component contributes $\simeq 45 \%-65 \%$ to the total unabsorbed $0.5-10 \mathrm{keV}$ model flux. These spectral parameters are typical for low-luminosity neutron star LMXBs in the hard state (e.g., Lin et al. 2009; Linares 2009; Armas Padilla et al. 2011). The average PC and WT outburst spectra are shown in Figure 2.

The corresponding $0.5-10 \mathrm{keV}$ model flux is $F_{X} \simeq 1.7 \times$ $10^{-10} \mathrm{erg} \mathrm{cm}^{-2} \mathrm{~s}^{-1}$, when averaged over the PC and WT data. For a distance of $D=3.7 \mathrm{kpc}$ (see Section 3.2), this translates into a mean outburst luminosity of $L_{X} \simeq 2.8 \times$ 


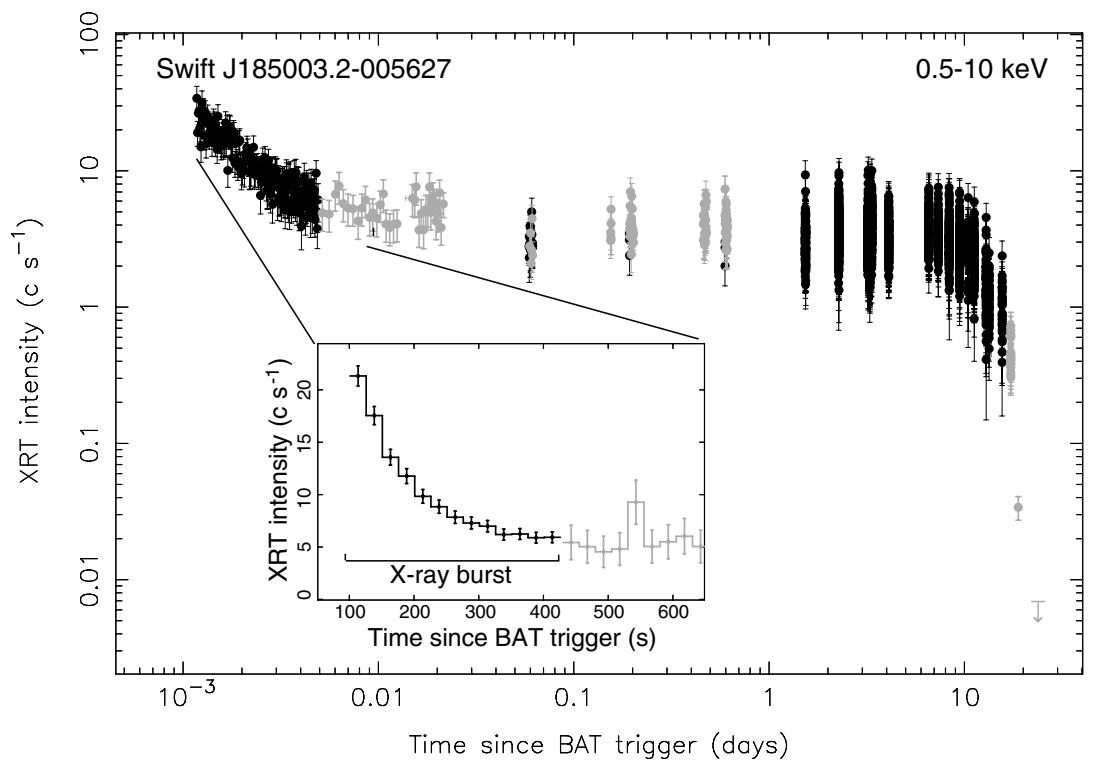

Figure 1. Swift/XRT 0.5-10 keV count rate light curves of J1850 using both WT (black) and PC (gray) data. The BAT trigger (No. 456041) occurred on 2011 June 24. The main image is a log-log plot showing the X-ray burst and subsequent outburst evolution using 10 counts per bin. The inset displays the X-ray burst light curve at $25 \mathrm{~s}$ resolution on a linear scale.

Table 2

Results from Spectral Fitting of the Averaged Outburst Data

\begin{tabular}{|c|c|c|c|c|c|c|c|c|}
\hline $\begin{array}{l}\text { Year } \\
\text { Data Mode }\end{array}$ & $\begin{array}{c}N_{\mathrm{H}} \\
\left(10^{22} \mathrm{~cm}^{-2}\right)\end{array}$ & $\Gamma$ & $\begin{array}{l}k T_{\mathrm{bb}} \\
(\mathrm{keV})\end{array}$ & $\begin{array}{l}R_{\mathrm{bb}} \\
(\mathrm{km})\end{array}$ & $\chi_{v}^{2}(\mathrm{dof})$ & $\begin{array}{c}F_{X} \\
\left(10^{-10} \mathrm{erg} \mathrm{cm}^{-2} \mathrm{~s}^{-1}\right)\end{array}$ & $\begin{array}{l}L_{X} \\
\left(10^{35} \mathrm{erg} \mathrm{s}^{-1}\right)\end{array}$ & $L_{\text {bol }}$ \\
\hline \multicolumn{9}{|c|}{ Swift J185003.2-005627 } \\
\hline 2011 & $1.1 \pm 0.1$ & & & & $1.1(669)$ & & & $\simeq 7$ \\
\hline WT & $\ldots$ & $1.6 \pm 0.2$ & $0.7 \pm 0.1$ & $1.8 \pm 0.2$ & $\ldots$ & $2.3 \pm 0.2$ & $3.8 \pm 0.3$ & $\ldots$ \\
\hline $\mathrm{PC}$ & $\ldots$ & $0.9 \pm 0.5$ & $0.7 \pm 0.1$ & $1.7 \pm 0.3$ & $\ldots$ & $1.1 \pm 0.1$ & $1.8 \pm 0.2$ & $\ldots$ \\
\hline \multicolumn{9}{|c|}{ Swift J1922.7-1716 } \\
\hline $2005-2006$ & $0.17 \pm 0.08$ & $\ldots$ & $\ldots$ & $\ldots$ & $0.9(209)$ & $\ldots$ & $\ldots$ & $\simeq 17$ \\
\hline WT & $\ldots$ & $1.5 \pm 0.4$ & $0.4 \pm 0.1$ & $6.6 \pm 0.7$ & $\ldots$ & $2.8 \pm 0.3$ & $7.7 \pm 0.8$ & $\ldots$ \\
\hline $\mathrm{PC}$ & $\ldots$ & $1.6 \pm 0.4$ & $0.4 \pm 0.1$ & $5.8 \pm 0.7$ & $\ldots$ & $2.2 \pm 0.3$ & $6.0 \pm 0.9$ & $\ldots$ \\
\hline 2011-2012 & $0.15 \pm 0.03$ & $\ldots$ & $\ldots$ & $\ldots$ & $1.0(660)$ & $\ldots$ & $\ldots$ & $\simeq 27$ \\
\hline WT & $\ldots$ & $1.7 \pm 0.1$ & $0.7 \pm 0.1$ & $3.8 \pm 0.4$ & $\ldots$ & $6.1 \pm 0.2$ & $16.8 \pm 0.5$ & $\ldots$ \\
\hline $\mathrm{PC}$ & $\ldots$ & $1.6 \pm 0.2$ & $0.5 \pm 0.1$ & $4.7 \pm 0.4$ & $\cdots$ & $1.7 \pm 0.1$ & $4.7 \pm 0.3$ & $\ldots$ \\
\hline
\end{tabular}

Notes. All quoted errors refer to $90 \%$ confidence levels. The outburst data were fitted to a combined power-law and blackbody model (POWERLAW+BBODYRAD), modified by absorption (PHABS). $F_{X}$ gives the total unabsorbed model flux in the $0.5-10 \mathrm{keV}$ band and $L_{X}$ represents the corresponding luminosity assuming distances of 3.7 and $4.8 \mathrm{kpc}$ for J1850 and J1922, respectively. $L_{\mathrm{bol}}$ represents the estimated bolometric accretion luminosity averaged over the outburst (see the text).

$10^{35} \mathrm{erg} \mathrm{s}^{-1}(0.5-10 \mathrm{keV})$. This allows for an estimate of the average bolometric accretion luminosity of $L_{\mathrm{bol}} \simeq 7 \times$ $10^{35}\left(D / 3.7 \mathrm{kpc}^{2} \mathrm{erg} \mathrm{s}^{-1}\right.$, which corresponds to $\simeq 0.2 \%$ of the Eddington limit (Section 2.4).

To investigate whether any spectral evolution occurred along the X-ray outburst, we compared the ratio of counts in the $2-10 \mathrm{keV}$ and $0.5-2 \mathrm{keV}$ energy bands (Figure 3 ). This suggests that the spectrum softens (i.e., the hardness ratio becomes smaller) as the intensity decreases. This behavior has been seen for several black hole and neutron star LMXBs transitioning from the hard state to quiescence (Armas Padilla et al. 2011 and references therein).

\subsubsection{UVOT}

Investigation of the UVOT images does not reveal a candidate optical/UV counterpart for J1850 (see also Beardmore et al. 2011a). This may not be surprising given the relatively high extinction in the direction of the source. Using the relation $N_{\mathrm{H}} / A_{\mathrm{V}}=(2.21 \pm 0.03) \times 10^{21}$ atoms cm $\mathrm{cmag}^{-1}$, the hydrogen column density inferred from spectral fitting $\left(N_{\mathrm{H}} \simeq 1.1 \times 10^{22} \mathrm{~cm}^{-2}\right)$, suggests an extinction of $A_{\mathrm{V}} \simeq 5.0$ mag in the $V$ band (Güver \& Özel 2009). Due to the proximity of a nearby bright source, we cannot obtain reliable upper limits for J1850 from the UVOT data.

We note that near-infrared (nIR) observations revealed a possible counterpart with $K \simeq 14.8$ mag within the $1^{\prime \prime} .7$ XRT positional uncertainty, although the probability of a chance detection is considerable in the crowded source region (Im et al. 2011). A weak optical source with $I \simeq 21$ mag was detected at a position consistent with the candidate nIR counterpart (Gorosabel et al. 2011).

\subsection{X-Ray Burst Properties}

\subsubsection{BAT Burst Peak}

Figure 4 displays the 15-25 keV BAT light curve of trigger 456014 at $2 \mathrm{~s}$ resolution. The light curve looks like a single peak centered at $t \simeq 2 \mathrm{~s}$ after the trigger. The source is visible for $\simeq 25 \mathrm{~s}$ from $\simeq 10 \mathrm{~s}$ prior to the BAT trigger to $\simeq 15 \mathrm{~s}$ thereafter. 


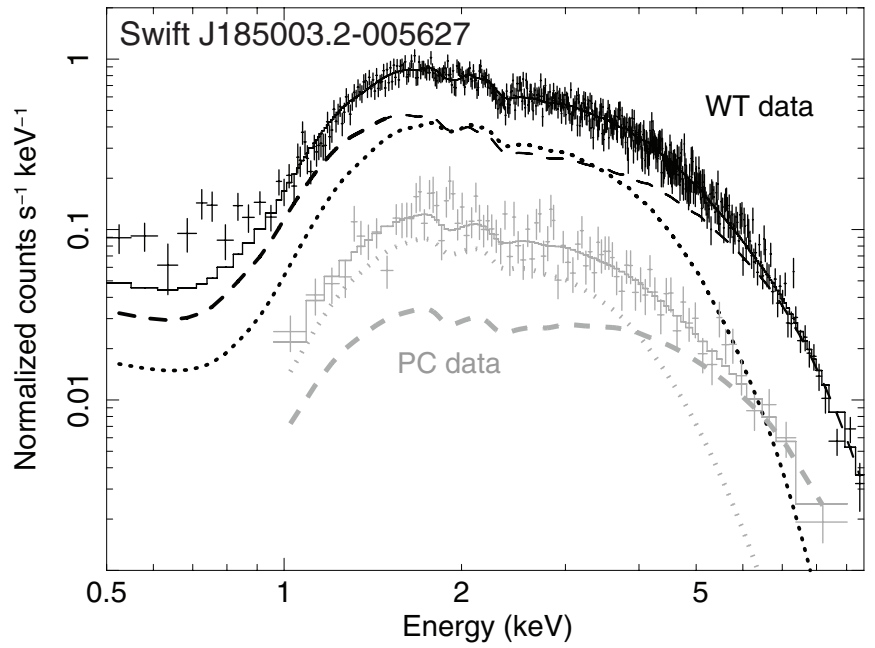

Figure 2. Swift/XRT spectra of the 2011 outburst of J1850, showing both WT (black) and PC (gray) data. The solid lines represent the best fit to a combined power-law (dashed line) and blackbody (dotted curve) model.

Slewing of the spacecraft started at $t \simeq 35 \mathrm{~s}$, when the source had already faded into the background. We used an interval of $20 \mathrm{~s}$ centered around the peak (covering $t=-5$ to $15 \mathrm{~s}$ ) to extract the average BAT spectrum.

The BAT spectrum is soft, with no source photons detected above $\simeq 35 \mathrm{keV}$. It fits to a blackbody with $k T_{\mathrm{bb}} \simeq 2.3 \mathrm{keV}$ and $R_{\mathrm{bb}} \simeq 5.6 \mathrm{~km}$, resulting in $\chi_{v}^{2}=1.0$ for 7 dof $(\operatorname{assuming} D=$ $3.7 \mathrm{kpc}$ and fixing $N_{\mathrm{H}}=1.1 \times 10^{22} \mathrm{~cm}^{-2}$ ). Extrapolating the model fit to the $0.01-100 \mathrm{keV}$ energy range yields an estimate of the bolometric flux of $F_{\text {bol }} \simeq 7.5 \times 10^{-8} \mathrm{erg} \mathrm{cm}^{-2} \mathrm{~s}^{-1}$. For a duration of $\simeq 20 \mathrm{~s}$, we estimate a (bolometric) fluence of $f_{\mathrm{BAT}} \simeq 1.5 \times 10^{-6} \mathrm{erg} \mathrm{cm}^{-2}$ (Table 3 ).

While the average count rate over the $20 \mathrm{~s}$ interval is $2.0 \times 10^{-2}$ counts $\mathrm{s}^{-1}$, the peak of the BAT data is a factor $\simeq 3$ higher. We therefore estimate a bolometric peak flux of $F_{\text {bol,peak }} \simeq 2.3 \times 10^{-7} \mathrm{erg} \mathrm{cm}^{-2} \mathrm{~s}^{-1}$. Assuming that the peak was equal to the empirical Eddington limit inferred from PRE bursts (Section 2.4) places the source at a distance of $D=3.7 \mathrm{kpc}$. The data do not reveal signatures of PRE, which implies that the burst may have been sub-Eddington. This distance estimate should therefore be regarded as an upper limit.

\subsubsection{XRT Burst Tail}

Observations with the narrow-field instruments commenced $\simeq 100 \mathrm{~s}$ after the BAT trigger. The inset of Figure 1 displays the XRT data, which shows a clear decay in count rate from $\simeq 20$ counts s$^{-1}$ at the start of the observation, leveling off to $\simeq 5$ counts s$^{-1}$ about $300 \mathrm{~s}$ later. This suggests that the total duration of the X-ray burst was $\simeq 400 \mathrm{~s}$ ( $\simeq 7$ minutes $)$.

The statistics of the XRT data do not allow for a detailed timeresolved spectroscopic analysis. To investigate whether the data exhibit the characteristic spectral softening seen in the tails of $\mathrm{X}$-ray bursts, we extracted two separate spectra for $t=101-201$ and $t=202-430 \mathrm{~s}$ after the burst trigger (all WT data).

We use an interval of $\simeq 500 \mathrm{~s}$ of PC data, obtained between $t=525$ and $1025 \mathrm{~s}$, to subtract the underlying accretion emission. Fitting this persistent spectrum to an absorbed powerlaw model yields an unabsorbed $0.5-10 \mathrm{keV}$ flux of $F_{X} \simeq$ $4.8 \times 10^{-10} \mathrm{erg} \mathrm{cm}^{-2} \mathrm{~s}^{-1}$. This suggests that $\mathrm{J} 1850$ was accreting at $\simeq 0.5 \%$ of the Eddington rate when the X-ray burst occurred.

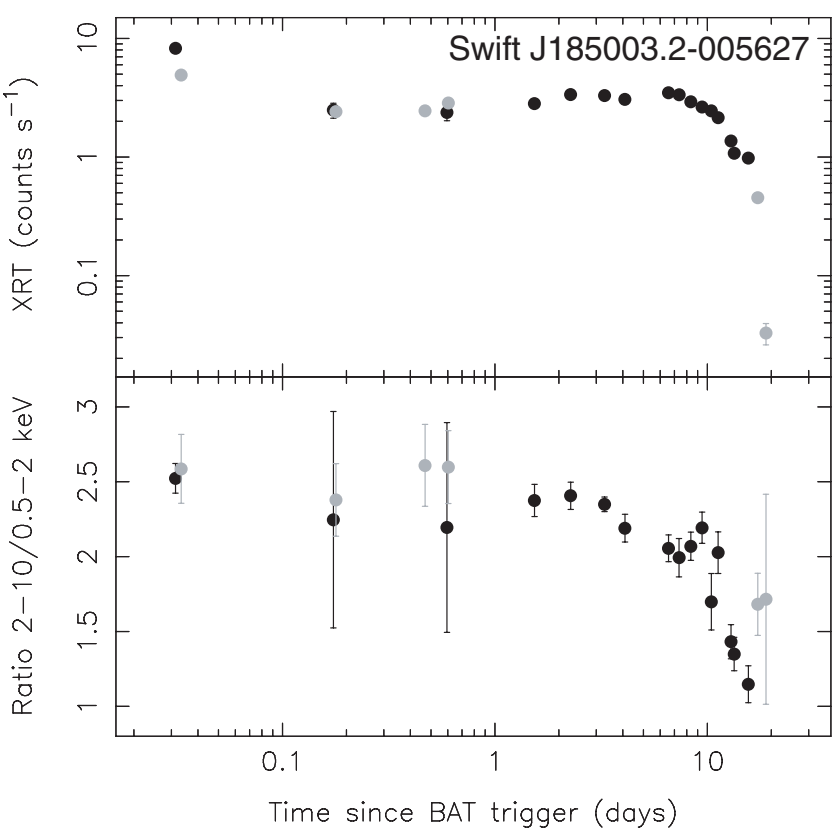

Figure 3. Evolution of the 2011 outburst of J1850 as seen with Swift/XRT. The black and gray points represent WT and PC mode data, respectively. The top plot shows the intensity in the full $(0.5-10 \mathrm{keV})$ energy band, whereas the bottom plot shows the ratio of counts in the hard (2-10 keV) and soft $(0.5-2 \mathrm{keV})$ bands.

The XRT spectra along the burst tail are best described by an absorbed blackbody model. We fix $N_{\mathrm{H}}=1.1 \times 10^{22} \mathrm{~cm}^{-2}$ (Section 3.1), and find that there is evidence of cooling along the $\simeq 300 \mathrm{~s}$ decay tail from $k T_{\mathrm{bb}} \simeq 0.81 \pm 0.03 \mathrm{keV}$ to $k T_{\mathrm{bb}} \simeq$ $0.73 \pm 0.03 \mathrm{keV}$, with corresponding blackbody emitting radii of $R_{\mathrm{bb}} \simeq 4-5 \mathrm{~km}$ (Table 3 ). The obtained spectral parameters are typical of X-ray burst tails and provide further support that the BAT was triggered by a thermonuclear event.

To investigate the shape of the decay, we fitted the XRT count rate light curve (binned by $10 \mathrm{~s}$ ) to a power law and an exponential function (Figure 5). For both decay functions, we include a constant offset to represent the underlying persistent $\mathrm{X}$-ray emission. The power-law fit yields a decay index of $\alpha=-2.1 \pm 0.1$ and a normalization of $\simeq 3.1 \times 10^{5}$ counts s$^{-1}$ $\left(\chi_{v}^{2}=0.7\right.$ for 30 dof $)$. For the exponential function, we obtain a decay time of $\tau=84.7 \pm 4.9 \mathrm{~s}$ and a normalization of $\simeq 54$ counts $^{-1}\left(\chi_{v}^{2}=0.5\right.$ for 30 dof $)$.

We apply a count rate to flux conversion factor inferred from fitting the average XRT burst spectrum and integrate both decay fits between $t=20 \mathrm{~s}$ (the time when the BAT intensity had returned to the background level) until $t=400 \mathrm{~s}$ (when the XRT intensity had leveled off to its persistent value). This yields an estimate of the fluence along the XRT burst tail of $f_{\mathrm{XRT}} \simeq(3.8-8.5) \times 10^{-7} \mathrm{erg} \mathrm{cm}^{-2}$. Adding this to the value obtained for the BAT data suggests a total fluence of $f_{\text {tot }} \simeq(1.9-2.4) \times 10^{-6} \mathrm{erg} \mathrm{cm}^{-2}$ for this event (which is corrected for the underlying persistent emission). For a distance of $D=3.7 \mathrm{kpc}$, the corresponding radiated energy is $E_{b} \simeq(3.1-3.9) \times 10^{39} \mathrm{erg}$.

We searched the light curves of all individual XRT observations for occurrences of X-ray bursts. Apart from the 2011 June 24 event, no other bursts were found.

\subsection{Quiescent Luminosity}

J1850 is within the field of view (FOV) of three archival XMM-Newton observations obtained in 2003 (Table 1). The source is not detected in any of these observations. We derive 

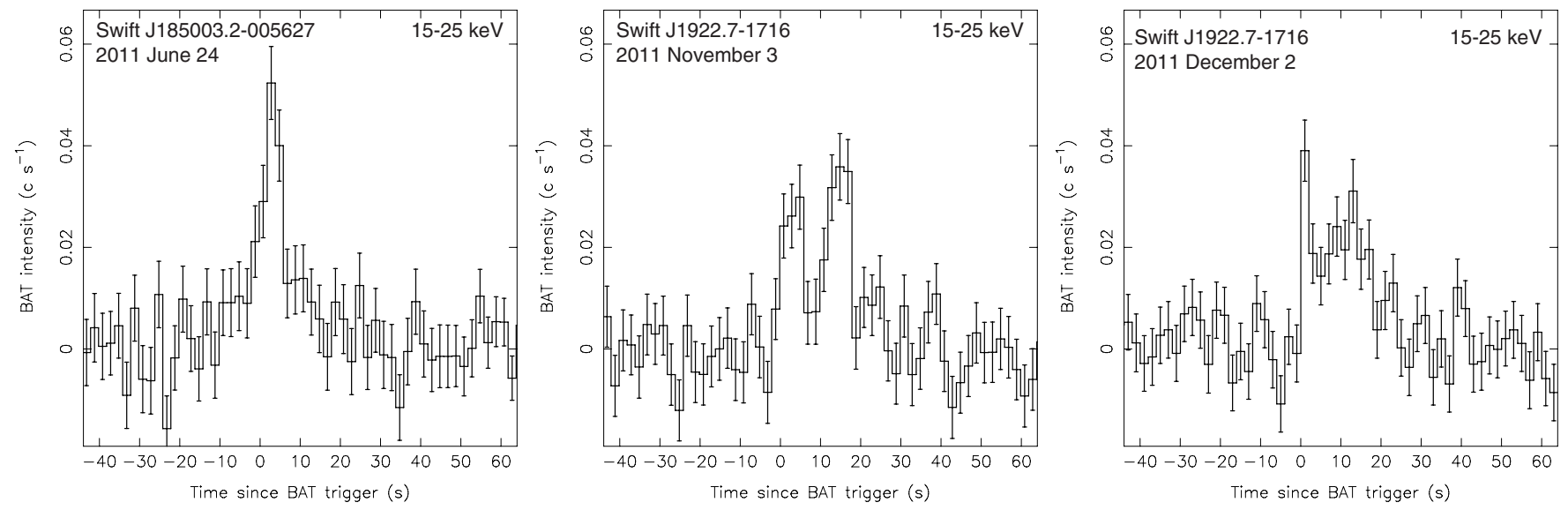

Figure 4. Swift/BAT light curve of trigger 456014 (left), 506913 (middle), and 508855 (right) at 2 s resolution (15-25 keV).

Table 3

Spectral Parameters of the X-Ray Bursts

\begin{tabular}{|c|c|c|c|c|c|c|}
\hline $\begin{array}{l}\text { Year } \\
\text { Data Mode }\end{array}$ & $\begin{array}{l}\Delta t \\
\text { (s) }\end{array}$ & $\begin{array}{c}N_{\mathrm{H}} \\
\left(10^{22} \mathrm{~cm}^{-2}\right)\end{array}$ & $\begin{array}{l}k T_{\mathrm{bb}} \\
(\mathrm{keV})\end{array}$ & $\begin{array}{c}R_{\mathrm{bb}} \\
(\mathrm{km})\end{array}$ & $\chi_{v}^{2}(\mathrm{dof})$ & $\begin{array}{c}F_{\mathrm{bol}} \\
\left(\mathrm{erg} \mathrm{cm}^{-2} \mathrm{~s}^{-1}\right)\end{array}$ \\
\hline \multicolumn{7}{|c|}{ Swift J185003.2-005627 } \\
\hline \multicolumn{7}{|c|}{2011 June 24} \\
\hline BAT & $0-20$ & $1.1 \mathrm{fix}$ & $2.3 \pm 0.6$ & $5.6_{-5.6}^{+6.5}$ & $1.0(7)$ & $(7.5 \pm 4.2) \times 10^{-8}$ \\
\hline XRT/WT & $101-201$ & $1.1 \mathrm{fix}$ & $0.81 \pm 0.03$ & $5.4 \pm 0.1$ & $1.1(66)$ & $(1.0 \pm 0.1) \times 10^{-9}$ \\
\hline XRT/WT & $202-430$ & $1.1 \mathrm{fix}$ & $0.73 \pm 0.03$ & $4.4 \pm 0.1$ & $1.2(68)$ & $(4.5 \pm 0.1) \times 10^{-10}$ \\
\hline \multicolumn{7}{|c|}{ Swift J1922.7-1716 } \\
\hline \multicolumn{7}{|c|}{2011 November 3} \\
\hline BAT & $0-20$ & 0.16 fix & $2.4 \pm 0.5$ & $6.7_{-6.7}^{+4.0}$ & $1.7(8)$ & $(6.8 \pm 3.2) \times 10^{-8}$ \\
\hline XRT/WT & $136-210$ & 0.16 fix & $0.80 \pm 0.07$ & $5.3 \pm 0.3$ & $0.9(84)$ & $(5.0 \pm 0.3) \times 10^{-10}$ \\
\hline XRT/WT & $211-440$ & 0.16 fix & $0.59 \pm 0.09$ & $5.0 \pm 0.6$ & $1.2(151)$ & $(1.4 \pm 0.1) \times 10^{-10}$ \\
\hline \multicolumn{7}{|c|}{2011 December 2} \\
\hline BAT & $0-20$ & 0.16 fix & $2.0 \pm 0.5$ & $14.9_{-14.9}^{+10.7}$ & $1.0(9)$ & $(10.2 \pm 6.3) \times 10^{-8}$ \\
\hline
\end{tabular}

Notes. All quoted errors refer to $90 \%$ confidence levels. The parameter $\Delta t$ indicates the time since the BAT trigger. The burst data were fitted to a blackbody model (BBODYRAD), modified by absorption (PHABS), with the hydrogen column density $\left(N_{\mathrm{H}}\right)$ fixed to the value obtained for the outburst fits. When calculating the emitting radii $\left(R_{\mathrm{bb}}\right)$, we assumed distances of 3.7 and $4.8 \mathrm{kpc}$ for J1850 and J1922, respectively. $F_{\mathrm{bol}}$ gives an estimate of the bolometric flux, which was obtained by extrapolating the blackbody fits to the $0.01-100 \mathrm{keV}$ energy range. We note that the emitting radii inferred from simple blackbody fits are expected to be underestimated due to the fact that electron scatterings in the neutron star atmosphere harden the spectrum resulting in a color temperature that is larger than the effective temperature by a factor $\simeq 1.5$ (e.g., Suleimanov et al. 2011). Consequently, the inferred radius is underestimated by that same factor.

a $95 \%$ confidence upper limit on the count rate of $\lesssim 1 \times$ $10^{-2}$ counts s$^{-1}$ for the PN data, and $\lesssim 1 \times 10^{-3}$ counts s $^{-1}$ for the MOS cameras, after applying the prescription for small numbers of counts given by Gehrels (1986). Using PIMMS (ver. 4.5), we estimate the corresponding upper limit on the quiescent luminosity.

Since the quiescent spectral shape is unknown, we explored both a power-law spectral model with $\Gamma=1-3$ and a blackbody of $k T_{\mathrm{bb}}=0.2-0.3 \mathrm{keV}$ (with $N_{\mathrm{H}}=1.1 \times 10^{22} \mathrm{~cm}^{-2}$ ), which are typical values found for the quiescent spectra of neutron star LMXBs (e.g., Asai et al. 1998; Rutledge et al. 1999; Wijnands et al. 2005a; Degenaar et al. 2012a). This results in an estimated upper limit on the $0.5-10 \mathrm{keV}$ quiescent luminosity of $L_{q} \lesssim(0.5-2.8) \times 10^{32}(D / 3.7 \mathrm{kpc})^{2} \mathrm{erg} \mathrm{s}^{-1}$.

\section{RESULTS FOR SWIFT J1922.7-1716}

\subsection{Outburst Properties}

\subsubsection{XRT}

Swift covered two different outbursts of J1922 in 2005-2006 and 2011-2012. The source was found active in all XRT observations that were carried out between 2005 July 8 and
2006 June 18. However, it is not detected with the XRT in a set of pointings taken between 2006 October 30 and November 14 . This demonstrates that the source had returned to quiescence (Section 4.3). The XRT data suggest that J1922 was active for at least 345 days in 2005-2006.

Hard X-ray monitoring data also indicate that the source experienced a long outburst. The BAT light curve $(15-150 \mathrm{keV})$ shows that it appeared active right at the start of the survey (2004 December; Tueller et al. 2005a, 2005b), until it faded $\simeq 20$ months later in 2006 July. Examination of the pre-processed INTEGRAL/IBIS-ISGR light curve of J1922 $(17-80 \mathrm{keV})$, which is publicly available via the High-Energy Astrophysics Virtually ENlightened Sky (HEAVENS; Lubiński 2009; Walter et al. 2010), ${ }^{8}$ suggests a similar outburst length as inferred from the BAT data. This is in agreement with the XRT results.

The average XRT spectrum of the 2005-2006 outburst can be described by a single absorbed power-law model, yielding $N_{\mathrm{H}} \simeq(2.9 \pm 0.3) \times 10^{21} \mathrm{~cm}^{-2}$ and $\Gamma=2.1 \pm 0.1\left(\chi_{v}^{2}=0.9\right.$ for 213 dof). Analysis of the broadband 2005 spectrum required

\footnotetext{
$8 \mathrm{http}: / / \mathrm{www}$. isdc.unige.ch/heavens/
} 


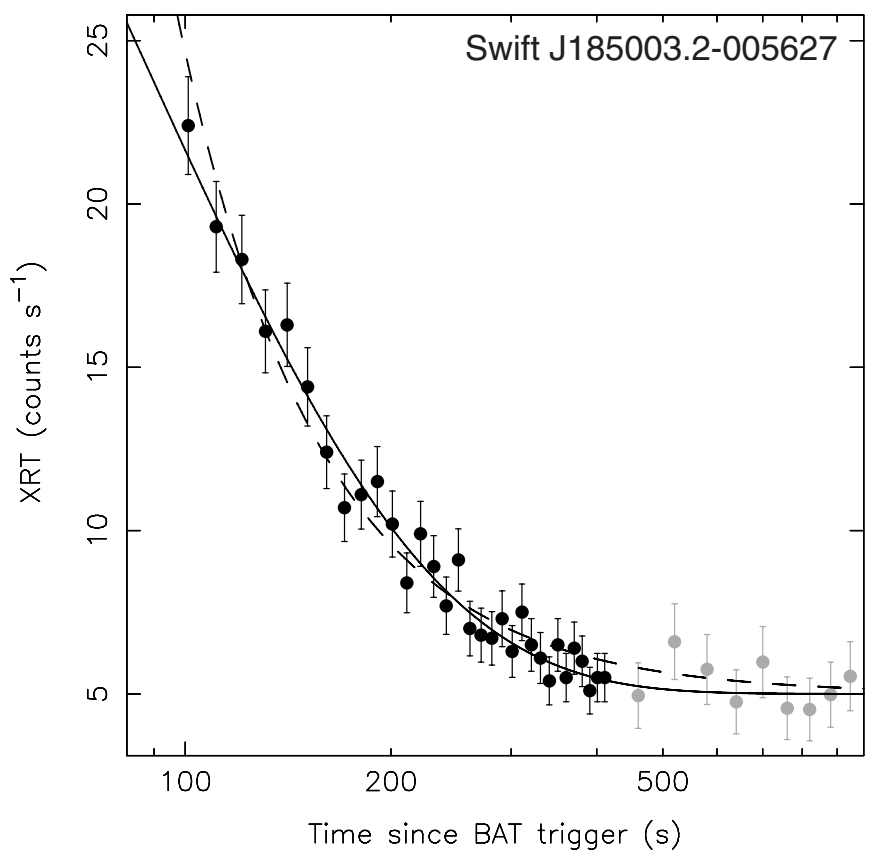

Figure 5. Swift/XRT tail of the X-ray burst of J1850 using a bin time of $10 \mathrm{~s}$ WT data are indicated in black, while PC data are colored gray. The full and dashed curves represent decay fits to an exponential and power-law function, respectively.

the addition of a soft emission component (Falanga et al. 2006). We obtain similar results for the 2011-2012 data (see below). Therefore, we included a blackbody component to the powerlaw fit for comparison.

The combined power-law and blackbody fit results in $N_{\mathrm{H}} \simeq$ $(1.7 \pm 0.1) \times 10^{21} \mathrm{~cm}^{-2}, \Gamma \simeq 1.5-1.6, k T_{\mathrm{bb}} \simeq 0.4 \pm 0.1 \mathrm{keV}$, $R_{\mathrm{bb}} \simeq 6-7 \mathrm{~km}$ (for $D=4.8 \mathrm{kpc}$ ), and $\chi_{v}^{2}=0.9$ for $209 \mathrm{dof}$ (Table 2). The blackbody component contributes $\simeq 20 \%$ to the total $0.5-10 \mathrm{keV}$ unabsorbed flux. These results are similar to those obtained for the broadband 2005 spectrum (Falanga et al. 2006).

The $0.5-10 \mathrm{keV}$ unabsorbed flux (averaged over the PC and WT data) for the combined model fit is $F_{X} \simeq 2.5 \times$
$10^{-10} \mathrm{erg} \mathrm{cm}^{-2} \mathrm{~s}^{-1}$. This translates into a mean outburst luminosity of $L_{X} \simeq 6.9 \times 10^{35} \mathrm{erg} \mathrm{s}^{-1}$ for a distance of $D=4.8 \mathrm{kpc}$ (Section 4.2). Assuming that the bolometric luminosity is a factor $\simeq 2.5$ higher than the intensity in the $0.5-10 \mathrm{keV}$ band, we estimate an average accretion luminosity of $L_{\text {bol }} \simeq$ $1.7 \times 10^{36}(D / 4.8 \mathrm{kpc})^{2} \mathrm{erg} \mathrm{s}^{-1}$, or $\simeq 0.5 \%$ of the Eddington limit (Section 2.4).

Swift/BAT and MAXI monitoring observations revealed that J1922 entered a new outburst around 2011 July 17 (Kennea et al. 2011). Between 2011 August 13 and 2012 March 15, J1922 was observed during a number of XRT pointings that all detected it in outburst. However, the source is no longer seen with the XRT on 2012 May 25 and follow-up observations performed between 2012 June 9 and 21 (Table 1). This suggests that the source had returned to quiescence (Figure 6 and Section 4.3). The 2011-2012 outburst had a duration of $\gtrsim 240$ days $(0.7 \mathrm{yr})$. If the Swift/BAT hard transient monitor caught the start of it, then the duration is constrained to $\lesssim 310$ days $(0.9 \mathrm{yr})$. The preprocessed publicly available IBIS-ISGR data do not cover this second outburst from J1922.

Fitting the average XRT spectrum of the 2011-2012 data with a simple absorbed power-law model yields $N_{\mathrm{H}} \simeq(2.7 \pm$ $0.1) \times 10^{21} \mathrm{~cm}^{-2}$ and $\Gamma \simeq 2.0 \pm 0.1\left(\chi_{v}^{2}=1.2\right.$ for $\left.664 \mathrm{dof}\right)$. The fit can be improved by adding a blackbody component, which results in $N_{\mathrm{H}} \simeq(1.5 \pm 0.1) \times 10^{21} \mathrm{~cm}^{-2}, \Gamma \simeq 1.6-1.7$, $k T_{\mathrm{bb}} \simeq 0.5-0.7 \mathrm{keV}, R_{\mathrm{bb}} \simeq 4-5 \mathrm{~km}$ (for $D=4.8 \mathrm{kpc}$ ), and $\chi_{v}^{2}=1.0$ for 660 dof (Table 2). The 2011-2012 spectral data and model fit are shown in Figure 7.

The best fit yields an average $0.5-10 \mathrm{keV}$ luminosity for the 2011-2012 outburst of $L_{X} \simeq 1.1 \times 10^{36}\left(D / 4.8 \mathrm{kpc}^{2} \mathrm{erg} \mathrm{s}^{-1}\right.$, and an estimated bolometric accretion luminosity of $L_{\mathrm{bol}} \simeq$ $2.7 \times 10^{36}(D / 4.8 \mathrm{kpc})^{2} \mathrm{erg} \mathrm{s}^{-1}(\simeq 1 \%$ of the Eddington limit). The blackbody component contributes $\simeq 20 \%$ to the total unabsorbed $0.5-10 \mathrm{keV}$ model flux. The spectral properties and intensity of the 2011-2012 outburst are comparable to that observed in 2005-2006 (Table 2).

Investigation of the ratio of counts in different energy bands (0.5-2 and 2-10 keV) does not reveal any evident spectral evolution along the outburst. It is of note that the dynamical range spanned by the observations of J1922 is narrower than

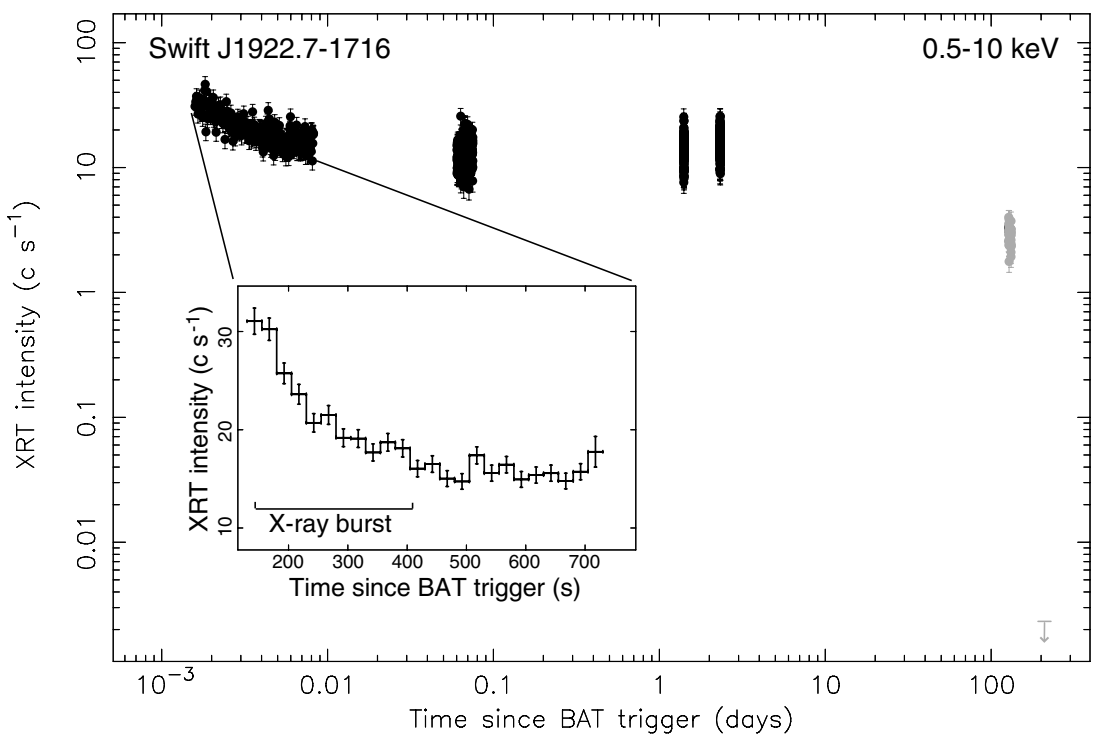

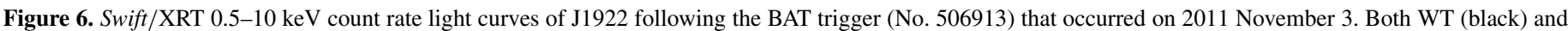

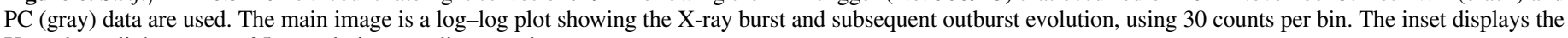
$\mathrm{X}$-ray burst light curve at $25 \mathrm{~s}$ resolution on a linear scale. 


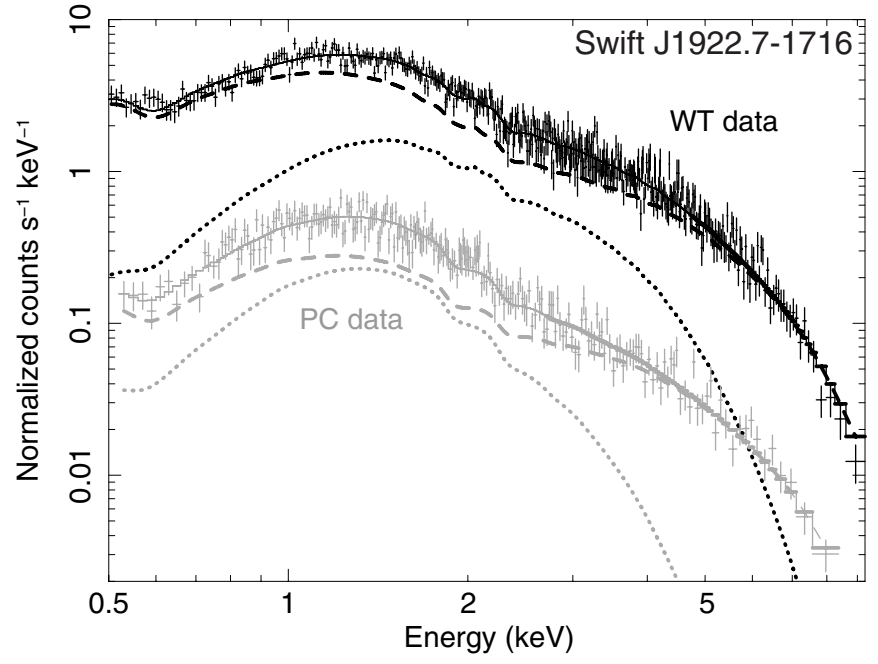

Figure 7. Swift/XRT spectra of the 2011-2012 outburst of J1922, showing both WT (black) and PC (gray) data. The solid lines represent the best fit to a combined power-law (dashed line) and blackbody (dotted curve) model.

that of J1850, for which the spectrum appeared to be softening with decreasing intensity (Section 3.1).

\subsubsection{UVOT}

Inspection of UVOT data reveals a possible counterpart at the position of J1922 in all filters (Table 4). This object is not present in Digitized Sky Survey (DSS) images (Barthelmy et al. 2011), and is not detected during the observations in which J1922 had faded into X-ray quiescence (2006 October-November and 2012 May-June; see Figures 8 and 9). This strongly suggests that the source detected in the UVOT images represents the optical/UV counterpart of J1922. As such, the UVOT data provide a subarcsecond localization of the LMXB (Barthelmy et al. 2011).

We have listed the magnitudes and upper limits of all UVOT observations in Table 4. These magnitudes are not corrected for interstellar extinction. Fits to the 2005-2006 and 2011-2012 XRT spectra yield $N_{\mathrm{H}} \simeq 1.6 \times 10^{21} \mathrm{~cm}^{-2}$, which suggests a visual extinction of $A_{\mathrm{V}} \simeq 0.7 \mathrm{mag}$ (Güver \& Özel 2009). The upper limits obtained with the UVOT for the quiescent state ( $v \gtrsim 20 \mathrm{mag}$, Table 4$)$ are consistent with the constraints from ground-based telescopes ( $>23.4$ mag in the $r$ and $g$ bands; Halpern \& Skinner 2011).

\subsection{X-Ray Burst Properties \\ 4.2.1. BAT Burst Peak}

Figure 4 displays the 15-25 keV BAT light curve of trigger 506913 at 2 s resolution. The light curve of J1922 starts rising $\simeq 2 \mathrm{~s}$ before the BAT trigger and shows two separate peaks at $t \simeq 5$ and $\simeq 15 \mathrm{~s}$. The source was visible in the BAT for $\simeq 25 \mathrm{~s}$ and had returned to the background level well before Swift started slewing ( $\simeq 65$ s post-trigger).

We extracted an average BAT spectrum using data from $t=0-20 \mathrm{~s}$, and fitted this spectrum between 15 and $35 \mathrm{keV}$. Using an absorbed blackbody model yields $k T_{\mathrm{bb}} \simeq 2.4 \mathrm{keV}$ and $R_{\mathrm{bb}} \simeq 6.7 \mathrm{~km}$ (for $D=4.8 \mathrm{kpc}$ and using $N_{\mathrm{H}}=1.6 \times$ $10^{21} \mathrm{~cm}^{-2}$; Table 3). Extrapolating the fit to the $0.01-100 \mathrm{keV}$ energy range results in an estimated average bolometric flux of $F_{\text {bol }} \simeq 6.8 \times 10^{-8} \mathrm{erg} \mathrm{cm}^{-2} \mathrm{~s}^{-1}$, and a fluence over the $20 \mathrm{~s}$ BAT interval of $f_{\mathrm{BAT}} \simeq 1.4 \times 10^{-6} \mathrm{erg} \mathrm{cm}^{-2}$. These spectral
Table 4

UVOT Observations and Results for J1922

\begin{tabular}{|c|c|c|c|c|}
\hline Obs ID & Date & Band & $\begin{array}{c}\text { Exp. } \\
(\mathrm{ks})\end{array}$ & Magnitude \\
\hline \multirow[t]{5}{*}{35174001} & 2005 Jul 8 & um2 & 1.7 & $16.13 \pm 0.03$ \\
\hline & & $u$ & 0.3 & $16.12 \pm 0.03$ \\
\hline & & $v$ & 0.5 & $17.11 \pm 0.06$ \\
\hline & & $u w 1$ & 1.1 & $16.05 \pm 0.03$ \\
\hline & & $u w 2$ & 2.3 & $16.05 \pm 0.03$ \\
\hline \multirow[t]{6}{*}{35174003} & 2005 Oct 1 & $b$ & & $17.31 \pm 0.04$ \\
\hline & & um 2 & 0.4 & $16.08 \pm 0.03$ \\
\hline & & $u$ & 2.7 & $16.17 \pm 0.03$ \\
\hline & & $v$ & 0.9 & $16.98 \pm 0.05$ \\
\hline & & $u w 1$ & 1.8 & $16.03 \pm 0.03$ \\
\hline & & $u w 2$ & 3.6 & $16.04 \pm 0.02$ \\
\hline 35471001 & 2006 Mar 14 & $u w 2$ & 1.5 & $17.62 \pm 0.03$ \\
\hline 35471002 & 2006 Jun 4 & $v$ & 0.3 & $17.45 \pm 0.10$ \\
\hline 35471003 & 2006 Jun 18 & $v$ & 1.0 & $17.42 \pm 0.05$ \\
\hline 35471004 & 2006 Oct 30 & $v$ & 5.1 & $>20.69$ \\
\hline 35471005 & 2006 Nov 3 & $v$ & 1.0 & $>19.74$ \\
\hline 35471006 & 2006 Nov 10 & $v$ & 4.2 & $>20.42$ \\
\hline 35471007 & 2006 Nov 14 & $v$ & 3.8 & $>20.24$ \\
\hline 35471008 & 2011 Aug 13 & um 2 & 1.0 & $16.52 \pm 0.04$ \\
\hline 35471009 & 2011 Aug 30 & $u w 1$ & 1.2 & $16.34 \pm 0.03$ \\
\hline 35471010 & 2011 Aug 31 & $u$ & 4.2 & $16.45 \pm 0.02$ \\
\hline \multirow[t]{7}{*}{506913000} & 2011 Nov 3 & $b$ & 0.2 & $17.13 \pm 0.06$ \\
\hline & & um2 & 0.2 & $17.80 \pm 0.06$ \\
\hline & & $u$ & 0.2 & $16.42 \pm 0.05$ \\
\hline & & $v$ & 0.2 & $17.40 \pm 0.14$ \\
\hline & & $u w 1$ & 0.2 & $17.64 \pm 0.05$ \\
\hline & & $u w 2$ & 0.2 & $16.03 \pm 0.05$ \\
\hline & & $w h$ & 0.4 & $16.64 \pm 0.04$ \\
\hline \multirow[t]{6}{*}{35471011} & 2011 Nov 4 & $b$ & 0.1 & $17.08 \pm 0.09$ \\
\hline & & um 2 & 0.2 & $15.98 \pm 0.06$ \\
\hline & & $u$ & 0.1 & $17.02 \pm 0.06$ \\
\hline & & $v$ & 0.1 & $17.54 \pm 0.28$ \\
\hline & & $u w 1$ & 0.1 & $15.97 \pm 0.06$ \\
\hline & & $u w 2$ & 0.3 & $15.85 \pm 0.04$ \\
\hline \multirow[t]{6}{*}{35471012} & 2011 Nov 5 & $b$ & 0.1 & $17.31 \pm 0.11$ \\
\hline & & um2 & 0.2 & $18.15 \pm 0.07$ \\
\hline & & $u$ & 0.1 & $16.44 \pm 0.08$ \\
\hline & & $v$ & 0.1 & $17.71 \pm 0.30$ \\
\hline & & $u w 1$ & 0.2 & $16.26 \pm 0.07$ \\
\hline & & $u w 2$ & 0.3 & $16.25 \pm 0.05$ \\
\hline \multirow[t]{2}{*}{35471013} & 2012 Mar 9 & $u$ & 0.4 & $17.12 \pm 0.07$ \\
\hline & & $u w 1$ & 0.3 & $17.11 \pm 0.09$ \\
\hline 35471014 & 2012 Mar 14 & $u$ & 1.0 & $17.08 \pm 0.04$ \\
\hline 35471015 & 2012 May 25 & $u$ & 1.2 & $>20.65$ \\
\hline 35471016 & 2012 Jun 9 & $u w 1$ & 1.9 & $>20.94$ \\
\hline 35471017 & 2012 Jun 11 & $u w 2$ & 2.0 & $>21.21$ \\
\hline 35471018 & 2012 Jun 13 & $u w 1$ & 0.9 & $>20.64$ \\
\hline 35471019 & 2012 Jun 15 & $u w 2$ & 0.7 & $>20.62$ \\
\hline 35471020 & 2012 Jun 17 & $u w 1$ & 0.3 & $>19.84$ \\
\hline 35471021 & 2012 Jun 21 & $u w 1$ & 2.3 & $>21.14$ \\
\hline
\end{tabular}

Notes. Quoted errors on the observed magnitudes correspond to a $1 \sigma$ confidence level. In the case of a non-detection, a $3 \sigma$ upper limit is given.

properties and energetics suggest that the BAT triggered on a thermonuclear event.

The BAT light curve shows a double-peaked structure (see also Barthelmy et al. 2011). This may be a signature of a PRE phase, suggesting that the burst reached the Eddington limit. However, the limited statistics prohibit confirmation via spectral analysis. The peak count rate seen by the BAT is a factor of $\simeq 2$ higher than the average count rate. We therefore estimate that the bolometric flux peaked at $F_{\text {bol,peak }} \simeq 1.4 \times 10^{-7} \mathrm{erg} \mathrm{cm}^{-2} \mathrm{~s}^{-1}$. 


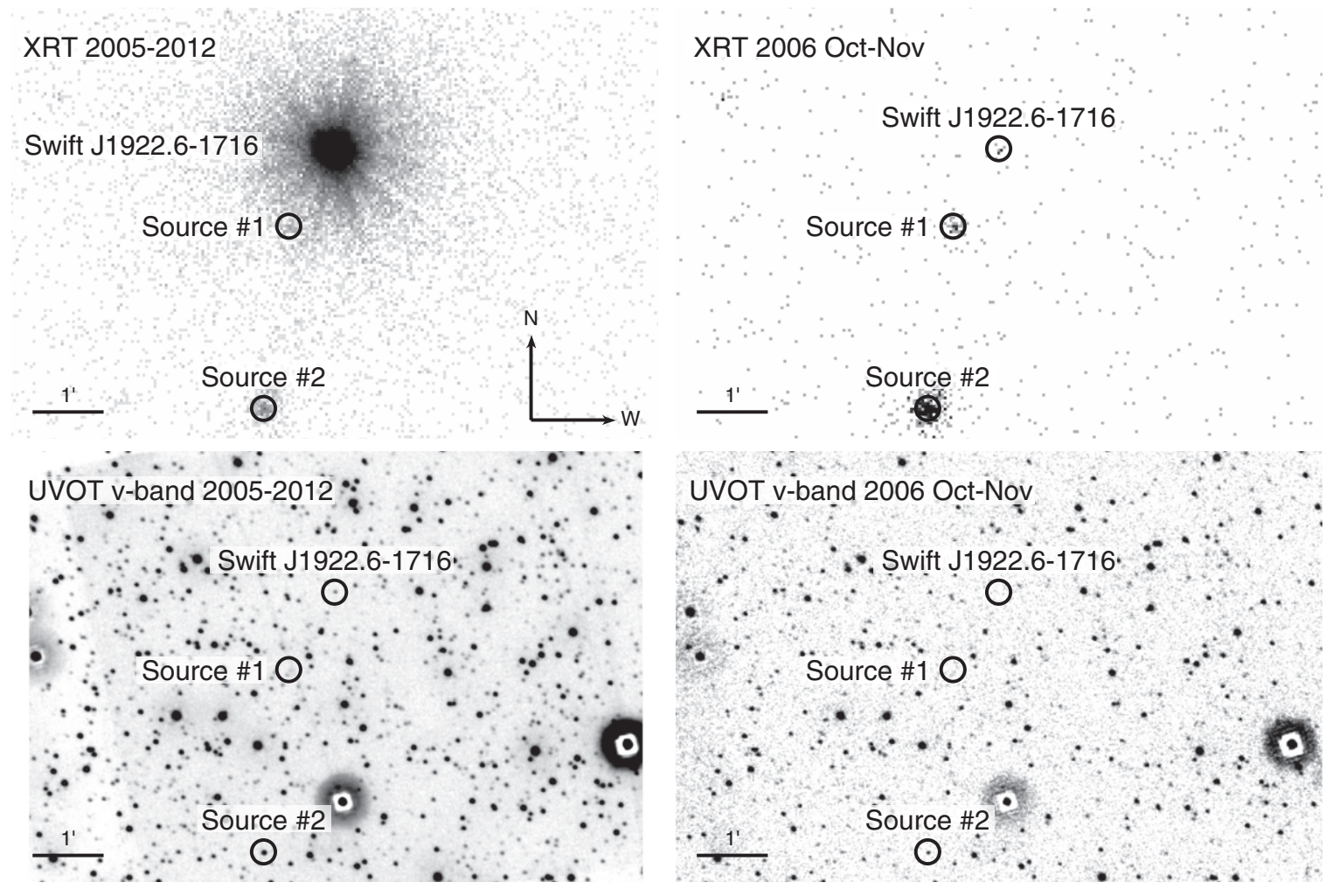

Figure 8. Images of the region around J1922. The location of J1922 and two nearby (uncataloged) X-ray sources are indicated by circles (see the Appendix for details on these serendipitous sources). Top left: accumulated image of all available Swift/XRT data (0.5-10 keV). Top right: Swift/XRT image of 2006 October-November. Bottom left: summed Swift/UVOT $v$-band image. Bottom right: Swift/UVOT $v$-band image of 2006 October-November.
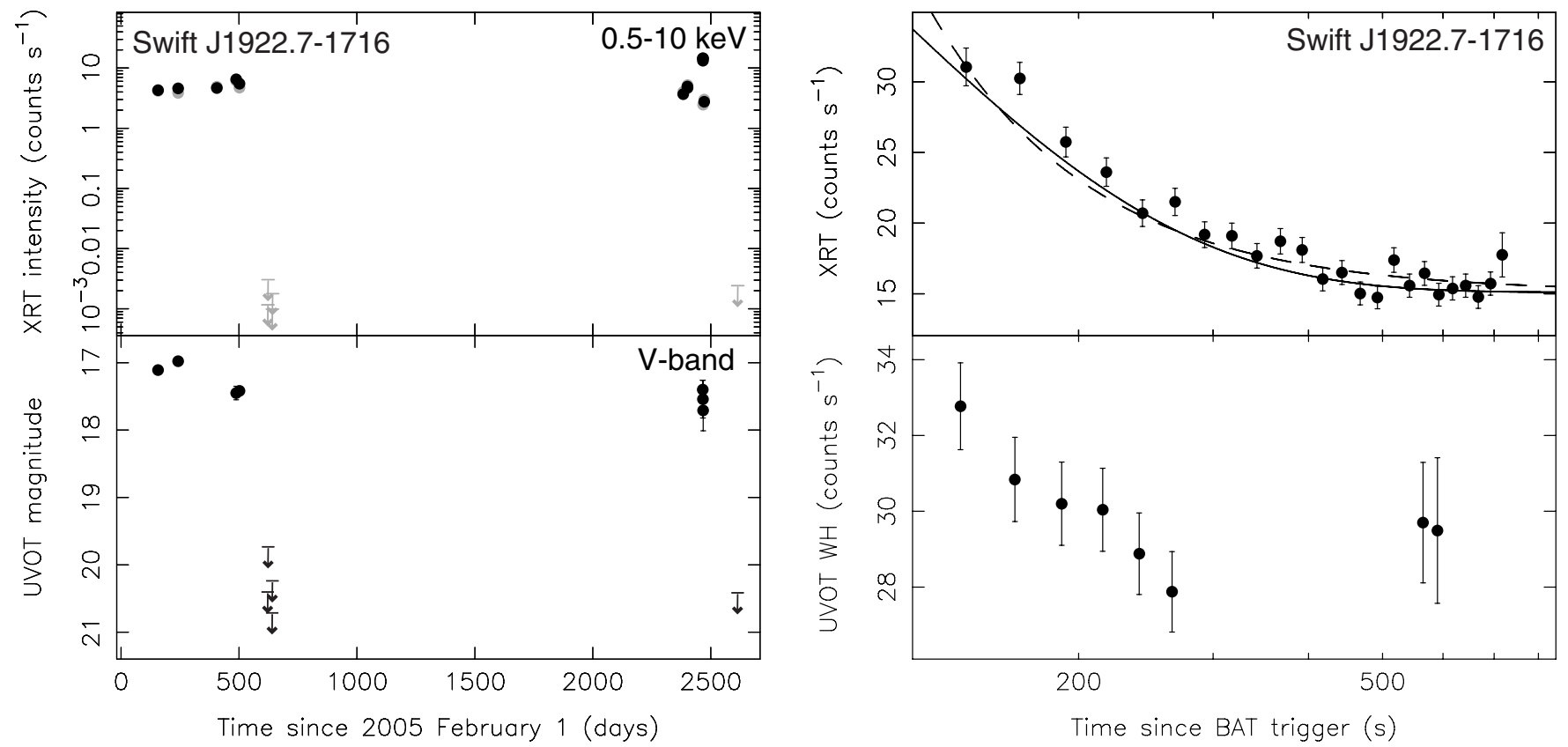

Figure 9. Left: Swift/XRT and UVOT $v$-band data of the two outbursts of J1922. For the XRT, WT data are indicated in black and PC data are colored gray. Right: Swift/XRT (WT mode) and UVOT $w$ h-band data of the follow-up observation of trigger 506913 (2011 November 3), showing the tail of the X-ray burst. The full and dashed curves represent an exponential and power-law decay fit, respectively.

If the peak reached the empirical Eddington limit of PRE bursts $\left(\simeq 3.8 \times 10^{38} \mathrm{erg} \mathrm{s}^{-1}\right.$; Kuulkers et al. 2003), then the source distance would be $D=4.8 \mathrm{kpc}$.

The BAT detected another event from the source region one month later on 2011 December 2 (trigger 508855). ${ }^{9}$

\footnotetext{
9 See http://gcn.gsfc.nasa.gov/notices_s/508855/BA/
}

The light curve of this trigger has a similar duration, intensity, and double-peaked structure as the one that occurred in 2011 November (Figure 4). We estimate a bolometric peak flux of $F_{\text {bol,peak }} \simeq 1.7 \times 10^{-7} \mathrm{erg} \mathrm{cm}^{-2} \mathrm{~s}^{-1}$ and a fluence of $f_{\mathrm{BAT}} \simeq 3.4 \times 10^{-6} \mathrm{erg} \mathrm{cm}^{-2}$. Given that the spectral properties and energetics of the two events are very similar (Table 3 ), 
we suggest that BAT trigger 508855 was also caused by an X-ray burst from J1922. No automated follow-up observations occurred for this trigger.

\subsubsection{XRT Burst Tail}

XRT follow-up observations commenced $\simeq 136 \mathrm{~s}$ after the BAT trigger of 2011 November 3. The XRT data were carried out in WT mode and show a clear decay in count rate during the first $\simeq 300 \mathrm{~s}$. The intensity peaks at the start of the observation at $\simeq 30$ counts $\mathrm{s}^{-1}$, after which it gradually decreases and levels off at $\simeq 15$ counts $\mathrm{s}^{-1}$. In subsequent observations, the count rate of $\mathrm{J} 1922$ remained at that level (Figure 6).

We extracted two separate spectra along the $300 \mathrm{~s}$ decay, and used the last $\simeq 165$ s of the observation as a background reference (all WT data; Figure 6). The spectra can be adequately fitted with a blackbody model that evolves from $k T_{\mathrm{bb}} \simeq$ $0.80 \pm 0.06 \mathrm{keV}$ in the first $75 \mathrm{~s}$ to $k T_{\mathrm{bb}} \simeq 0.59 \pm 0.09 \mathrm{keV}$ in the subsequent $225 \mathrm{~s}$ of the decay (for $N_{\mathrm{H}}=1.6 \times 10^{21} \mathrm{~cm}^{-2}$; Table 3). Such temperatures are typical for the cooling tail of an X-ray burst, further supporting the idea that the BAT triggered on a thermonuclear event. Investigation of the postburst persistent spectrum yields a $0.5-10 \mathrm{keV}$ unabsorbed flux of $F_{X} \simeq 7.8 \times 10^{-10} \mathrm{erg} \mathrm{cm}^{-2} \mathrm{~s}^{-1}$. This suggests that $\mathrm{J} 1922$ was accreting at $\simeq 1.4 \%$ of the Eddington rate when the burst occurred.

Based on the XRT data, we estimate a total duration for the X-ray burst of $\simeq 400 \mathrm{~s}$ (see Figure 6 ). The count rate light curve can be described by a power law with a decay index of $\alpha=-2.1 \pm 0.2$ and a normalization of $\simeq 4.1 \times 10^{6}$ counts s $^{-1}$ $\left(\chi_{v}^{2}=1.7\right.$ for $\left.25 \mathrm{dof}\right)$, or an exponential with a decay time of $\tau=102 \pm 9 \mathrm{~s}$ and a normalization of $\simeq 62$ counts $^{-1}\left(\chi_{v}^{2}=1.6\right.$ for 25 dof). For both decay fits, we included a constant offset to subtract the accretion emission.

We estimate the fluence in the XRT burst tail by applying a count rate to flux conversion factor inferred from fitting the average XRT burst spectrum and integrating the two different decay fits from $t=20 \mathrm{~s}$ (the time when the BAT intensity had faded to the background) until $t=400 \mathrm{~s}$ since the trigger (the time when the XRT count rate had leveled off). This yields $f_{\mathrm{XRT}} \simeq(5.4-9.7) \times 10^{-7} \mathrm{erg} \mathrm{cm}^{-2}$ for the burst tail (which is corrected for the underlying persistent emission). Combined with the BAT, this suggests a total burst fluence of $f_{\text {tot }} \simeq(1.9-2.4) \times 10^{-6} \mathrm{erg} \mathrm{cm}^{-2}$. This corresponds to a radiated energy of $E_{b} \simeq(5.4-6.5) \times 10^{39}$ erg for $D=4.8 \mathrm{kpc}$.

We note that there are two other X-ray sources located within the XRT FOV of J1922 (Figure 8). We briefly discuss the properties of these serendipitous X-ray sources in the Appendix. Since the XRT data following BAT trigger 506913 were taken only in WT mode, we cannot formally exclude one of these other $\mathrm{X}$-ray objects as sources of the BAT trigger based on the X-ray data alone. However, we investigated the simultaneous UVOT data of the trigger observation and found that the intensity of J1922 in the UVOT $w h$ band was decreasing simultaneously with the decay seen in the XRT data (Figure 9). This provides strong evidence that it was indeed J1922 that triggered the BAT. We did not find any other occurrences of X-ray bursts in the XRT data.

\subsection{Quiescent Luminosity}

J1922 went undetected during four consecutive XRT observations carried out between 2006 October 30 and November 14 (Obs IDs 35471004-7). We obtain count rate upper limits for these individual exposures of $\lesssim(1-3) \times 10^{-3}$ counts $\mathrm{s}^{-1}$
$(0.5-10 \mathrm{keV})$. Summing the four observations $(\simeq 13.8 \mathrm{ks}$ in total) reveals a small excess of 11 photons at the source position (see Figure 8), whereas five photons are expected from a set of three randomly placed background regions. The inferred count rate is $4.3 \times 10^{-4}$ counts s${ }^{-1}$. For $N_{\mathrm{H}}=1.6 \times 10^{21} \mathrm{~cm}^{-2}$, a power-law model with $\Gamma=1-3$, or a blackbody with $k T_{\mathrm{bb}}=0.2-0.3 \mathrm{keV}$, we estimate a $0.5-10 \mathrm{keV}$ quiescent luminosity of $L_{q} \simeq(0.4-0.9) \times 10^{32}(D / 4.8 \mathrm{kpc})^{2} \mathrm{erg} \mathrm{s}^{-1}$.

We note that this quiescent detection occurred within $\simeq 4$ months after the end of a long $(\simeq 2 \mathrm{yr}$ ) accretion outburst of $\mathrm{J} 1922$. It is possible that the neutron star became considerably heated during this prolonged outburst and did not yet cool down at the time of the Swift/XRT observations (Section 6.2). In this case, the true quiescent level could be lower than inferred here.

Inspection of an archival $78.6 \mathrm{ks}$ long Suzaku observation, performed on 2007 April 10, reveals a possible weak detection at the position of J1922 in the combined XIS image. In the source region, a total of $\simeq 450$ photons are detected, whereas $\simeq 350$ are expected from the background. We estimate a source count rate of $\simeq 1.3 \times 10^{-3}$ counts $\mathrm{s}^{-1}$. Using the range of spectral parameters stated above, we obtain a $0.5-10 \mathrm{keV}$ luminosity of $L_{q} \simeq(0.6-1.0) \times 10^{32}(D / 4.8 \mathrm{kpc})^{2} \mathrm{erg} \mathrm{s}^{-1}$. This is comparable to our estimate obtained from the $S$ wift/XRT observations.

On 2012 May 25, the source was not detected during a single Swift/XRT observation with an upper limit on the count rate of $\lesssim 2.5 \times 10^{-3}$ counts $^{-1}$ at $95 \%$ confidence level (Gehrels 1986). This translates into a $0.5-10 \mathrm{keV}$ quiescent luminosity upper limit $L_{q} \lesssim(2.2-2.5) \times 10^{32}(D / 4.8 \mathrm{kpc})^{2} \mathrm{erg} \mathrm{s}^{-1}$, and strongly suggests that the 2011-2012 outburst had ceased.

We obtained a series of Swift follow-up observations between 2012 June 9 and 21 (Table 1). The source is not detected in the combined XRT image (total exposure time of $\simeq 9.2 \mathrm{ks}$ when including the observation of 2012 May 25). We infer an upper limit on the count rate of $\lesssim 6.8 \times 10^{-4}$ counts s $^{-1}$ (95\% confidence; Gehrels 1986). Using the range of spectral parameters given above, we obtain a $0.5-10 \mathrm{keV}$ upper limit of $L_{q} \lesssim(0.4-1.4) \times 10^{32}(D / 4.8 \mathrm{kpc})^{2} \mathrm{erg} \mathrm{s}^{-1}$.

\section{BURST OSCILLATION SEARCH}

Several neutron star LMXBs have shown nearly coherent (millisecond) oscillations during X-ray bursts that measure the spin period of the neutron star (for reviews, see Strohmayer \& Bildsten 2006; Watts 2012). We searched the 0.5-10 keV Swift/XRT data of the X-ray burst tails for coherent pulsations in the frequency range $1-250 \mathrm{~Hz}$ (restricted by the time resolution of the data), using Fourier techniques. We first computed and searched Leahy-normalized (Leahy et al. 1983) power spectra throughout each burst using $16 \mathrm{~s}$ independent segments. This revealed no significant signal.

To estimate upper limits, we used the burst averaged power spectra calculated from a set of Leahy-normalized power spectra with a $1 \mathrm{~s}$ window. This takes into account possible frequency drifts (within $1 \mathrm{~Hz}$ ) and assumes that coherent oscillations are present during the whole burst. We followed Vaughan et al. 1994, and searched for the largest observed power in the $0.5-10 \mathrm{keV}$ band. Using the Groth-distribution (Groth 1975), we obtain upper limits of $\simeq 3 \%$ and $\simeq 2 \%$ fractional rms amplitude for J1850 and J1922, respectively (99.7\% confidence level). If we assume that the oscillations are only present during the first $\simeq 5 \mathrm{~s}$ sampled with the XRT, then the upper limits for both sources are unconstrained $(\gtrsim 20 \%)$. 
Table 5

Summary of X-Ray Burst Properties

\begin{tabular}{|c|c|c|}
\hline Parameter & Swift J185003.2-005627 & Swift J1922.7-1716 \\
\hline Exponential decay time, $\tau(\mathrm{s})$ & $\simeq 85$ & $\simeq 102$ \\
\hline Total duration, $t_{\mathrm{b}}(\mathrm{s})$ & $\simeq 400$ & $\simeq 400$ \\
\hline Total fluence, $f_{\text {tot }}\left(\mathrm{erg} \mathrm{cm}^{-2}\right)$ & $\simeq(1.9-2.4) \times 10^{-6}$ & $\simeq(1.9-2.4) \times 10^{-6}$ \\
\hline Distance, $D(\mathrm{kpc})$ & $\lesssim 3.7$ & $\lesssim 4.8$ \\
\hline Post-burst accretion luminosity, $L_{\text {bol }}\left(\mathrm{erg} \mathrm{s}^{-1}\right)$ & $\lesssim 2 \times 10^{36}$ & $\lesssim 5 \times 10^{36}$ \\
\hline Post-burst mass-accretion rate, $\dot{M}\left(M_{\odot} \mathrm{yr}^{-1}\right)$ & $\widetilde{\leqslant}_{2} \times 10^{-10}$ & $\widetilde{\Sigma}_{4} \times 10^{-10}$ \\
\hline
\end{tabular}

Notes. The rise time refers to that seen in the BAT. The burst duration is estimated as the time from the BAT trigger until the flux decayed back to the persistent level as observed with the XRT. The quoted peak fluxes are unabsorbed and for the $0.01-100 \mathrm{keV}$ energy range. The quoted accretion luminosity and mass-accretion rate were inferred from fitting $\simeq 200-500$ s of post-burst persistent emission. The burst listed for Swift J1922.7-1716 is the one that triggered the BAT in 2011 November, for which follow-up XRT observations were carried out. We note that the shape of X-ray bursts can look very different in different energy bands, so caution should be taken when comparing rise and decay times seen with different instruments.

The low count rates in the Swift/BAT data (Figure 4) did not allow us to obtain a meaningful upper limits on the occurrence of oscillations during the peak of the X-ray bursts.

\section{DISCUSSION}

We investigated three Swift/BAT triggers that occurred in 2011. The BAT trigger spectra are soft and can be described by a blackbody model with a temperature of $k T_{\mathrm{bb}} \simeq 2-3 \mathrm{keV}$. Rapid follow-up XRT observations were obtained for two of the triggers and revealed a decaying X-ray intensity that levels off to a constant value $\simeq 400 \mathrm{~s}$ after the BAT peak. The $X$-ray tail shows a soft (thermal) emission spectrum that cools during the decay to $k T_{\text {bb }} \simeq 0.6-0.7 \mathrm{keV}$. Both events have a comparable exponential decay time $(\tau \simeq 85-100 \mathrm{~s})$, fluence $\left(f_{\text {total }} \simeq 2 \times 10^{-6} \mathrm{erg} \mathrm{cm}^{-2}\right)$, and radiated energy output $\left(E_{b} \simeq 3-7 \times 10^{39} \mathrm{erg}\right.$; Table 5). These features are consistent with thermonuclear bursts occurring on accreting neutron stars, and strongly suggest that the BAT triggers were caused by type-I X-ray bursts.

We identified the X-ray transients J1850 and J1922 as the origin of the BAT triggers. This implies that both sources are neutron star LMXBs. J1850 is a previously unknown X-ray source, whereas J1922 was already discovered in 2005 but remained unclassified (Falanga et al. 2006). We characterize the outburst and quiescent properties of these two new X-ray bursters.

The outburst of J1850 seen with Swift's BAT and XRT had a duration of $\simeq 8$ weeks, but the onset of the outburst is not well constrained and hence it may have lasted (considerably) longer. The average $0.5-10 \mathrm{keV}$ intensity of $L_{X} \simeq 3 \times$ $10^{35}(D / 3.7 \mathrm{kpc})^{2} \mathrm{erg} \mathrm{s}^{-1}$ classifies the source as a very-faint $\mathrm{X}$-ray transient (Table 2; cf. Wijnands et al. 2006). At the time of the X-ray burst, the source was accreting at $\simeq 0.5 \%$ of the Eddington rate. Investigation of the ratio of XRT counts in different energy bands as the outburst progressed revealed that the spectrum softens when the intensity decreases. This behavior has been seen in several neutron star and black hole LMXBs transitioning from the hard state to quiescence, although the underlying mechanism is not understood well (see Armas Padilla et al. 2011, for a recent example and an overview).

J1922 exhibited an outburst in 2005-2006 with an observed duration of $\simeq 20$ months and an $0.5-10 \mathrm{keV}$ intensity of $L_{X} \simeq$ $7 \times 10^{35}(D / 4.8 \mathrm{kpc})^{2} \mathrm{erg} \mathrm{s}^{-1}$. The $2011-2012$ outburst likely commenced in 2011 mid-July and had ceased by 2012 May, implying a duration of $\simeq 8-10$ months. The $0.5-10 \mathrm{keV}$ luminosity of this second outburst was $L_{X} \simeq 1 \times 10^{36}(D / 4.8 \mathrm{kpc})^{2} \mathrm{erg} \mathrm{s}^{-1}$. These outburst intensities fall in the very faint to faint regime (Table 2; cf. Wijnands et al. 2006). At the time of the BAT trigger of 2011 November, the source was accreting at $\simeq 1.4 \%$ of the Eddington limit. Using Swift/UVOT data, we have identified a unique UV/optical counterpart to J1922. This provides a subarcsecond localization of the source (Barthelmy et al. 2011).

\subsection{X-Ray Burst Properties of J1850 and J1922}

The observable properties of X-ray bursts (e.g., the duration, recurrence time, and radiated energy) depend on the conditions of the ignition layer, such as the thickness, temperature, and $\mathrm{H}$-abundance. These can drastically change as the massaccretion rate onto the neutron star varies, such that there exist distinct accretion regimes that give rise to X-ray bursts with different characteristics (Fujimoto et al. 1981; Bildsten 1998). The overall similarities between the properties of the X-ray bursts of J1850 and J1922 (Table 5) suggest that these events were ignited under similar conditions.

In the accretion regime of $\mathrm{J} 1850$ and $\mathrm{J} 1922(\simeq 0.5 \%-1 \%$ of Eddington), the temperature of the burning layer is expected to be low enough for $\mathrm{H}$ to burn unstably. This may in turn trigger He ignition in an H-rich environment, which typically results in $\simeq 10-100 \mathrm{~s}$ long bursts (e.g., Fujimoto et al. 1981; Bildsten 1998; Galloway et al. 2008). The detection of H-emission lines in the optical spectrum of J1922 suggests that the neutron star is accreting H-rich material (Wiersema et al. 2011; Halpern \& Skinner 2011). This implies that the fuel triggering the X-ray burst could have indeed contained H. Given the similarities in burst properties, J1850 may therefore be expected to host an H-rich companion star as well.

The observed burst duration of $t_{b} \simeq 400 \mathrm{~s}(\tau \simeq 85-100 \mathrm{~s})$ is considerably longer than that typically observed for H-rich X-ray bursts (Chenevez et al. 2008; Linares et al. 2012). The burst profiles may look very different depending on the energy band in which they are observed (Chelovekov et al. 2006; Linares et al. 2012), and most bursts known to date have been detected with instruments that cover higher energies than Swift/XRT (typically $>2 \mathrm{keV}$, e.g., RXTE, INTEGRAL, BeppoSAX, Fermi; Cornelisse et al. 2003; Chenevez et al. 2008; 
Galloway et al. 2008; Linares et al. 2012). However, there are several X-ray bursts observed with Swift/XRT, Chandra, or $X M M$-Newton (i.e., in the same energy band as the bursts observed from J1850 and J1922) from neutron star LMXBs accreting at $\simeq 1 \%$ of Eddington that do show the expected duration of $\simeq 10-100$ s (e.g., Boirin et al. 2007; Trap et al. 2009; Degenaar \& Wijnands 2009; Degenaar et al. 2012b).

The uncommon properties of the bursts from J1850 and J1922 are further illustrated by their energetics. The estimated radiated energies of $E_{b} \lesssim(3-7) \times 10^{39}$ erg are higher than those typically found for normal X-ray bursts, yet lower than those classified as intermediately long (Chenevez et al. 2008; Linares et al. 2012). There are several possible explanations that may account for the unusual burst properties.

First, for some LMXBs, the first X-ray burst that occurred during a new outburst was found to be significantly longer than later bursts (e.g., Aql X-1 and Cen X-4; Fushiki et al. 1992; Kuulkers et al. 2009). It is thought that at the start of the outburst the neutron star is relatively cold and therefore a thicker layer of fuel can build up before the ignition conditions are met. Since the duration of an X-ray burst depends on the cooling time of the ignition layer, a thicker layer would result in a longer (and more energetic) X-ray burst. By considering the expected burst recurrence time, we can assess whether this scenario might be applicable to J1850 and J1922.

With the burst energetics at hand, the ignition depth can be estimated as $y=E_{b}(1+z) / 4 \pi R^{2} Q_{\text {nuc,burst }}$, where $z$ is gravitational redshift, $R$ is the neutron star radius, and $Q_{\text {nuc,burst }}=$ $1.6+4 X \mathrm{MeV}$ nucleon ${ }^{-1}$, the nuclear energy release during an $\mathrm{X}$-ray burst given an $\mathrm{H}$-fraction $X$ at ignition (e.g., Galloway et al. 2008). For a neutron star with $M=1.4 M_{\odot}$ and $R=10 \mathrm{~km}$ (i.e., $z=0.31$ ), we estimate $y \simeq 10^{-8} \mathrm{~g} \mathrm{~cm}^{-2}$ for the bursts of $\mathrm{J} 1850$ and $\mathrm{J} 1922$. The recurrence time that corresponds to a given ignition depth is $t_{\text {rec }} \simeq y(1+z) / \dot{m}$, where $\dot{m}$ is the accretion rate onto the neutron star surface per unit area. For J1850 and $\mathbf{J} 1922$, we infer an average accretion rate during outburst of $\dot{m}=\dot{M} / 4 \pi R^{2} \simeq 300$ and $\simeq 2300 \mathrm{~g} \mathrm{~cm}^{-2} \mathrm{~s}^{-1}$, respectively (assuming that the emission is isotropic). This would imply an expected recurrence time on the order of a few days to two weeks.

The BAT transient monitor revealed activity from J1850 $\simeq 5$ weeks before the BAT trigger of 2011 June 24 . J1922 had been accreting for $\simeq 3$ months prior to its BAT trigger on 2011 November 3, as evidenced by Swift and MAXI (Sections 1.1 and 1.2). Comparing this to the expected burst recurrence time of $\lesssim 2$ weeks suggests that the BAT triggers were likely not the first X-ray bursts that occurred during the outbursts of J1850 and J1922. The BAT coverage starts at $15 \mathrm{keV}$, which implies that it is not optimally sensitive to detect events as soft as X-ray bursts. These are therefore easily missed.

A second scenario that is worth considering is that some $\mathrm{X}$-ray bursts display prolonged tails that last up to an hour (e.g., EXO 0748-676 and the "clocked burster" GS 1826-24; in 't Zand et al. 2009). These are explained as the cooling of layers below the ignition layer that were heated by inward conduction of energy generated during the X-ray burst. These long cooling tails are characterized by fluxes and fluences that are two orders of magnitude lower than the prompt burst emission. In the case of J1850 and J1922, however, the fluence in the BAT peak and XRT tail are of similar magnitude. This indicates that the long duration of these X-ray bursts is likely caused by a different mechanism.

Alternatively, the long burst duration may be explained in terms of a relatively large $\mathrm{H}$-content in the ignition layer.
The presence of $\mathrm{H}$ lengthens the duration of nuclear energy generation via the rp-process (Schatz et al. 2001). There are two additional effects that likely contribute in making the bursts observable for a longer time. At low accretion rates, the temperature in the neutron star envelope is expected to be low compared to brighter bursters. This implies that a thicker fuel layer can accumulate before reaching the critical ignition conditions, resulting in a longer burst. Furthermore, J1850 and $\mathrm{J} 1922$ accreted at a relatively low level of $\simeq 0.5 \%-1 \%$ of Eddington. For such low persistent emission levels, the tails of the X-ray bursts are visible for a longer time (if observed with a sensitive instrument), which can add to a longer burst duration. The burst recurrence time at the accretion rates inferred for $\mathrm{J} 1850$ and J1922 is considerably lower than for higher rates. This can account for the fact that most observed X-ray bursts are shorter than those seen for these two sources.

Intermediately, long X-ray bursts are typically observed from sources accreting at $\simeq 0.1 \%-1 \%$ of Eddington. These events are both longer ( $\gtrsim 10$ minutes) and more energetic $\left(\simeq 10^{40-41} \mathrm{erg}\right)$ than we have observed for J1850 and J1922 (e.g., in 't Zand et al. 2008; Falanga et al. 2009; Linares et al. 2009; Degenaar et al. 2011b). Some of these sources are strong candidate ultra-compact X-ray binaries. These contain an H-depleted companion star so that the neutron star accretes (nearly) pure He (e.g., in 't Zand et al. 2008). In absence of H-burning, the temperature in the accreted envelope is relatively low, so that a thick layer of He can build up before it eventually ignites in a long and energetic X-ray burst. A similarly long X-ray burst has also been observed, however, from a source that accretes at $\simeq 0.1$ of Eddington and shows a strong $\mathrm{H}$-emission line in its optical spectrum. This testifies to the presence of an H-rich companion (Degenaar et al. 2010). In this case, it may be that unstable H-burning could not immediately trigger He ignition, allowing the development of a thick layer of fuel (Cooper \& Narayan 2007; Peng et al. 2007).

To summarize, the burst duration and energetics of the X-ray bursts observed from J1850 and J1922 appear to fall in between that of normal and intermediately long X-ray bursts (cf. Chenevez et al. 2008; Linares et al. 2012). Similar bursts have also been observed from the persistent neutron star LMXB 4U 0614+09 (Kuulkers et al. 2010; Linares et al. 2012). We considered several possible scenarios that may account for the relatively long burst duration, and find it most likely that it is due to the ignition of a relatively thick layer of (H-rich) fuel. Our findings suggests that there may not exist two distinct groups of $\mathrm{X}$-rays bursts, but rather a continuous range of burst durations and energies. This supports the idea proposed by Linares et al. (2012) that the apparent bimodal distribution is likely due to an observational bias toward detecting the longest and most energetic X-ray bursts from slowly accreting neutron stars.

\subsection{Quiescent Properties of J1850 and J1922}

J1850 could not be detected in archival XMM-Newton observations with an estimated upper limit of $L_{q} \lesssim(0.5-3.0) \times$ $10^{32}(D / 3.7 \mathrm{kpc})^{2} \mathrm{erg} \mathrm{s}^{-1} . \mathrm{J} 1922$ is detected in quiescence with Swift/XRT and Suzaku at a $0.5-10 \mathrm{keV}$ luminosity of $L_{q} \simeq$ $(0.4-1.0) \times 10^{32}(D / 4.8 \mathrm{kpc})^{2} \mathrm{erg} \mathrm{s}^{-1}$. These quiescent levels are common for neutron star LMXBs (e.g., Menou et al. 1999; Garcia et al. 2001; Jonker et al. 2004).

It is thought that the accretion of matter onto the surface of a neutron star compresses the stellar crust and induces a chain of nuclear reactions that deposit heat (Haensel \& Zdunik 1990). This heat spreads over the entire stellar body via thermal 
conduction and maintains the neutron star at a temperature that is set by the long-term averaged accretion rate of the binary (Brown et al. 1998; Colpi et al. 2001).

During quiescent episodes, the neutron star is expected to thermally emit X-rays providing a candescent luminosity of $L_{\mathrm{q}, \text { bol }}=\langle\dot{M}\rangle Q_{\mathrm{nuc}} / m_{u}$, where $Q_{\text {nuc }} \simeq 2 \mathrm{MeV}$ is the nuclear energy deposited in the crust per accreted baryon (Haensel \& Zdunik 2008; Gupta et al. 2007, but see Degenaar et al. 2011a), $m_{u}=1.66 \times 10^{-24} \mathrm{~g}$ is the atomic mass unit (i.e., $Q_{\text {nuc }} / m_{u} \simeq$ $\left.9.6 \times 10^{17} \mathrm{erg} \mathrm{g}^{-1}\right)$, and $\langle\dot{M}\rangle$ is the long-term accretion rate of the binary averaged over $\simeq 10^{4} \mathrm{yr}$ (i.e., including both outburst and quiescent episodes). The latter can be estimated by multiplying the average accretion rate observed during outburst $\left(\dot{M}_{\mathrm{ob}}\right)$ with the duty cycle of the binary (i.e., the ratio of the outburst duration and recurrence time).

The outburst of J1850 observed with Swift in 2011 had a duration of $\simeq 8$ weeks $(0.17 \mathrm{yr})$ and an estimated bolometric accretion luminosity of $L_{\text {bol }} \simeq 7 \times 10^{35}(D / 3.7 \mathrm{kpc})^{2} \mathrm{erg} \mathrm{s}^{-1}$ (corresponding to $\dot{M}_{\mathrm{ob}} \simeq 6 \times 10^{-11} M_{\odot} \mathrm{yr}^{-1}$ ). The outburst duration and recurrence time are not known for $\mathrm{J} 1850$, but we can make an order of magnitude estimate based on the constraints of the quiescent luminosity.

Within the deep crustal heating model, a quiescent luminosity of $\lesssim 3 \times 10^{32}(D / 3.7 \mathrm{kpc})^{2} \mathrm{erg} \mathrm{s}^{-1}$ would suggest a timeaveraged mass-accretion rate of $\langle\dot{M}\rangle \lesssim 5 \times 10^{-12} M_{\odot} \mathrm{yr}^{-1}$. For $\dot{M}_{\mathrm{ob}} \simeq 6 \times 10^{-11} M_{\odot} \mathrm{yr}^{-1}$, the corresponding duty cycle is $\lesssim 10 \%$. If J1850 typically exhibits outbursts with a duration of 8 weeks, then the expected recurrence time would be $\gtrsim 2 \mathrm{yr}$. We regard this as a lower limit, since the expected recurrence time increases for a longer outburst duration, or a quiescent thermal luminosity that is lower than the assumed upper limit of $3 \times 10^{32} \mathrm{erg} \mathrm{s}^{-1}$. This estimate is consistent with the fact that the Swift/BAT hard transient monitor did not detect any other outbursts from J1850 back to 2005 February (Krimm et al. 2011).

Since two outbursts with a relatively well-constrained duration have been observed for J1922, we can reverse the above reasoning and estimate the quiescent luminosity that is expected based on the observed duty cycle. The source was discovered when it exhibited an outburst of $\simeq 20$ months in 2005-2006. Renewed activity was observed from the source 5 yr later in 2011-2012, when it accreted for $\simeq 8-10$ months. For both outbursts, we estimate a similar bolometric accretion luminosity of $L_{\text {bol }} \simeq(2-3) \times 10^{36} \mathrm{erg} \mathrm{s}^{-1}$, which suggests a mean mass-accretion rate of $\dot{M}_{\mathrm{ob}} \simeq 3 \times 10^{-10} M_{\odot} \mathrm{yr}^{-1}$ (Table 2). If we assume a typical outburst duration of $\simeq 1.3 \mathrm{yr}$ and a recurrence time of $\simeq 5$ yr (i.e., a duty cycle of $\simeq 26 \%$ ), then we can estimate a long-term averaged mass-accretion rate of $\langle\dot{M}\rangle \simeq 1 \times 10^{-10} M_{\odot} \mathrm{yr}^{-1}$ (equivalent to $\simeq 6 \times 10^{15} \mathrm{~g} \mathrm{~s}^{-1}$ ).

If the outburst behavior observed over the past decade is typical for the long-term accretion history of J1922, then the estimated $\langle\dot{M}\rangle$ should give rise to $L_{\mathrm{q}, \text { bol }} \simeq 6 \times$ $10^{33}(D / 4.8 \mathrm{kpc})^{2} \mathrm{erg} \mathrm{s}^{-1}$. This is considerably higher than the observed $L_{q} \simeq 1 \times 10^{32}(D / 4.8 \mathrm{kpc})^{2} \mathrm{erg} \mathrm{s}^{-1}(0.5-10 \mathrm{keV})$. Although the bolometric luminosity may be a factor of a few higher than that measured in the $0.5-10 \mathrm{keV}$ band, the luminosity remains lower than expected based on the crustal heating model. Limited by a low number of counts, we cannot constrain the shape of the quiescent spectrum of J1922 with the current available data. It is possible that the quiescent emission contains a significant non-thermal component (e.g., Campana et al. 2005; Wijnands et al. 2005b; Heinke et al. 2009; Cackett et al. 2010b; Degenaar \& Wijnands 2012; Degenaar et al. 2012a), which would increase the discrep- ancy between the expected and observed quiescent thermal emission.

Provided that the heating models are correct, a plausible explanation for this apparent mismatch could be that the timeaveraged mass-accretion rate is lower than estimated (e.g., because the recent outburst behavior is not representative for the long-term accretion history). Alternatively, the neutron star could be cooling faster than assumed in the standard paradigm (Page et al. 2004). A comparison with theoretical cooling models of Yakovlev \& Pethick (2004) would suggest enhanced cooling due to the presence of kaons in the neutron star core.

Four neutron star LMXBs have been closely monitored after the cessation of their very long ( $\gtrsim 1 \mathrm{yr}$ ) accretion outbursts, which has revealed that the neutron star temperature was gradually decreasing over the course of several years (Wijnands et al. 2001, 2003; Cackett et al. 2008, 2010a; Degenaar et al. 2009, 2011c; Fridriksson et al. 2010, 2011; Díaz Trigo et al. 2011). Recently, similar behavior has been observed for a transient neutron star LMXB in Terzan 5 that exhibited a much shorter accretion outburst of $\simeq 10$ weeks (Degenaar $\&$ Wijnands 2011; Degenaar et al. 2011a). These observations can be explained as cooling of the neutron star crust, which became considerably heated during the accretion outburst and needs time to cool down in quiescence. Monitoring and modeling this crustal cooling provides the unique opportunity to gain insight into the properties of the neutron star crust and core (Rutledge et al. 2002; Wijnands 2005; Shternin et al. 2007; Brown \& Cumming 2009; Degenaar et al. 2011a; Page \& Reddy 2012).

With its relatively low quiescent luminosity, long outburst duration, and low extinction, J1922 would be a promising target to search for crustal cooling now that its recent outburst has ceased.

N.D. is supported by NASA through Hubble Postdoctoral Fellowship grant number HST-HF-51287.01-A from the Space Telescope Science Institute, which is operated by the Association of Universities for Research in Astronomy, Incorporated, under NASA contract NAS5-26555. R.W. is supported by a European Research Council (ERC) starting grant, and M.L. by a Rubicon fellowship from the Netherlands Organization for Scientific Research (NWO). N.D. thanks Min-Su Shin for useful discussion about flaring M-stars. This work made use of data supplied by the UK Swift Science Data Centre at the University of Leicester and the public data archives of Swift, XMM-Newton, and Suzaku.

Facilities: Swift (BAT/XRT/UVOT), XMM (EPIC), Suzaku (XIS)

\section{APPENDIX}

\section{SERENDIPITOUS FAINT X-RAY SOURCES}

The XRT images of the field around J1922 reveal two additional faint X-ray sources (Figure 8). Neither of these objects are cataloged in the SIMBAD database. We assigned them Swift names and determined their positions and basic properties (Table 6). We used the tools XRTCENTROID and UVOTCENTROID to determine their positions.

Source 1 is located in the wing of the point spread function of J1922, and is most clearly visible when the transient is in quiescence (see Figure 8). To avoid contamination, we extracted information for source 1 using only observations 35471004-7 (when J1922 was not active; Table 1). The object is detected at an average XRT count rate of $\simeq 1 \times 10^{-3}$ counts s $^{-1}(0.5-10 \mathrm{keV})$. 

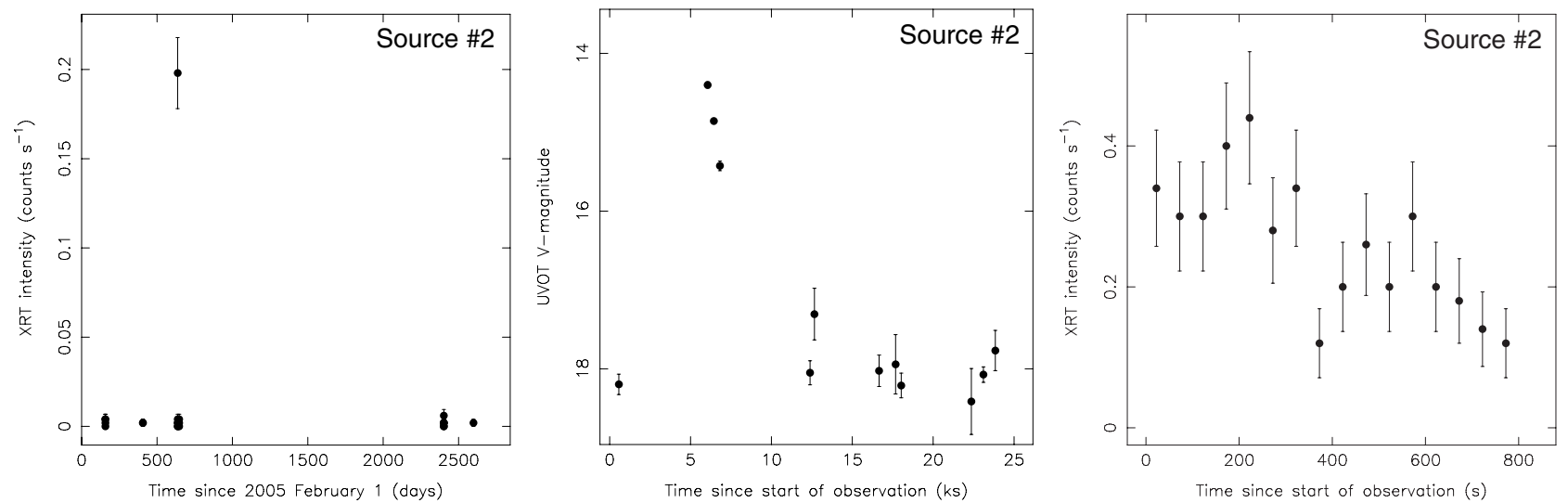

Figure 10. Results for source 2 (Swift J192241.1-272040) that was serendipitously detected within FOV of J1922. Left: XRT light curve obtained between 2005 and 2012 (binned per orbit). Middle: evolution of the UVOT $v$-band magnitude during the flare observation of 2006 October 30. Right: XRT light curve of the orbit containing the flare (50 $\mathrm{s}$ resolution).

Table 6

Source Properties

\begin{tabular}{lcccccc}
\hline \hline Source No. & Name & $\begin{array}{c}\text { R.A. } \\
(\mathrm{hh} \mathrm{mm} \mathrm{ss.ss})\end{array}$ & $\begin{array}{c}\text { Decl. } \\
\left({ }^{\circ}{ }^{\prime \prime}\right)\end{array}$ & $\begin{array}{c}\text { Error } \\
\left({ }^{\prime \prime}\right)\end{array}$ & $\begin{array}{c}\text { XRT Count Rate } \\
\left(10^{-3} \text { counts s }^{-1}\right)\end{array}$ & $\begin{array}{c}\text { UVOT } v \text {-magnitude } \\
(\mathrm{mag})\end{array}$ \\
\hline 1 & Swift J192239.5-171808 & 192239.54 & -171808.0 & 3.8 & $\simeq 1$ & $\simeq 2-19.4-20.3$ \\
2 & Swift J192241.1-272040 & 192241.15 & -172039.4 & 0.7 & $\simeq 2-250$ & $14.4-18.2$ \\
\hline
\end{tabular}

Notes. The listed coordinates refer to the J2000 epoch. Errors represent a 90\% confidence level. The XRT count rates are for the $0.5-10 \mathrm{keV}$ energy band.

There are not enough counts collected to extract an X-ray spectrum. DSS and UVOT images reveal a possible counterpart at the XRT position (Table 6). This object is detected in some of the UVOT images (at 3.3-12.2 $\sigma$ significance) with $v \simeq$ 19.4-20.3 mag. We derive a position of R.A. = 19:22:39.61, decl. $=-17: 18: 08.3(\mathrm{~J} 2000)$, with a $90 \%$ confidence uncertainty of 0.9 . For other observations, we obtain upper limits of $v>18.8-20.2 \mathrm{mag}$. The intensity is close to the detection limit of the UVOT observations.

Source 2 is detected at an average XRT count rate of $\simeq 2 \times 10^{-3}$ counts $^{-1}$, but experienced an X-ray flare that was a factor $\simeq 100$ brighter on 2006 October 30 (Obs ID 35471004; Figure 10, left). This observation had a total exposure time of $\simeq 5.2 \mathrm{ks}$ and consisted of five separate orbits. The enhanced activity is detected in the second orbit, which had an exposure time of $\simeq 850 \mathrm{~s}$. During this interval, the source intensity decreased from $\simeq 0.4$ to $\simeq 0.1$ counts s$^{-1}$, but remained well above $\simeq 10^{-3}$ counts $s^{-1}$ (Figure 10, right). In both the preceding and subsequent orbits, the source is detected at its persistent count rate, which implies that the X-ray flare had a total duration between 0.25 and $3 \mathrm{hr}$.

We extracted X-ray spectra of both the flare and the persistent emission. We fitted these simultaneously to an absorbed powerlaw model with the hydrogen column density tied between the two data sets. This resulted in $N_{\mathrm{H}}=(1.8 \pm 0.2) \times 10^{21} \mathrm{~cm}^{-2}$ and $\chi_{v}^{2}=1.1$ for 9 dof (Figure 11). The persistent X-ray spectrum can be described with $\Gamma=1.5 \pm 0.7$, while the flare appears to be softer with $\Gamma=2.1 \pm 0.5$. The unabsorbed $0.5-10 \mathrm{keV}$ fluxes are $(1.2 \pm 0.3) \times 10^{-13}$ and $(1.7 \pm 0.5) \times 10^{-11} \mathrm{erg} \mathrm{cm}^{-2} \mathrm{~s}^{-1}$ for the persistent and flare emission, respectively.

DSS and UVOT images reveal a possible counterpart centered at the XRT position of source 2 (Figure 8). We find magnitudes of $v \simeq 17.6-18.2 \mathrm{mag}$ for the persistent emission of this object, while it brightened up to $v \simeq 14.4 \mathrm{mag}$ during the second orbit of the flare observation. Figure 10 (middle) displays the evolution of the UVOT $v$-magnitude during that observation.

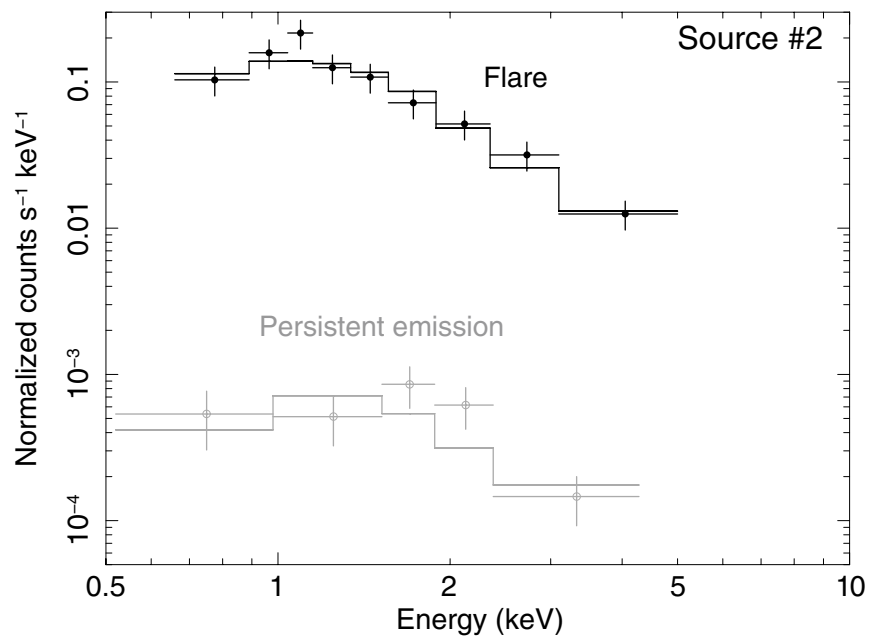

Figure 11. Swift/XRT spectra of source 2 for the flare (black) and persistent (gray) emission, together with the best-fit absorbed power-law model.

The simultaneous brightening in the optical and X-ray bands firmly establishes that the object seen in the UVOT images is indeed the counterpart to source 2 . We obtained a subarcsecond localization from the UVOT data (Table 6).

There are two observations in which source 2 is detected in both the $b$ and the $v$ band, which allows for a color determination. This gives $(b-v)=1.36-1.46 \mathrm{mag}$. The source is not detected with the $u$-filter on these occasions, which implies $(u-b)>0.9$. Comparing these colors and the observed $v$-magnitude to stars of different spectral classes (Drilling \& Landolt 2000), we find that the UVOT object could be an M-dwarf located at a distance of $D \simeq 0.4 \mathrm{kpc}$ (where we have taken into account the visual extinction in the direction of the source of $A_{\mathrm{V}} \simeq 1 \mathrm{mag}$, as determined using the relation of Güver \& Özel 2009).

M-dwarfs are the primary stellar component in the Galaxy by number (Bochanski et al. 2010) and are known to exhibit 
flares that are visible across the electromagnetic spectrum (e.g., Hawley et al. 2003). At a distance of $0.4 \mathrm{kpc}$, the observed persistent emission and that of the flare translate into luminosities of $\simeq 2 \times 10^{30}$ and $\simeq 2 \times 10^{32} \mathrm{erg} \mathrm{s}^{-1}$, respectively. These intensities, as well as the observed duration and amplitude of the flare, are consistent with the properties of flaring M-dwarfs (e.g., Hawley et al. 2003). We therefore tentatively classify source 2 , Swift J192241.1-272040, as a nearby Galactic M-dwarf.

\section{REFERENCES}

Anders, E., \& Grevesse, N. 1989, Geochim. Cosmochim. Acta, 53, 197 Armas Padilla, M., Degenaar, N., Patruno, A., et al. 2011, MNRAS, 417, 659 Arnaud, K. A. 1996, in ASP Conf. Ser. 101, Astronomical Data Analysis Software and Systems V, ed. G. H. Jacoby \& J. Barnes (San Francisco, CA: ASP), 17

Asai, K., Dotani, T., Hoshi, R., et al. 1998, PASJ, 50, 611

Balucinska-Church, M., \& McCammon, D. 1992, ApJ, 400, 699

Barthelmy, S. D., Barbier, L. M., Cummings, J. R., et al. 2005, Space Sci. Rev., 120,143

Barthelmy, S. D., Baumgartner, W. H., Burrows, D. N., et al. 2011, GRB Coordinates Network, 12522

Beardmore, A. P., Baumgartner, W. H., Gelbord, J. M., et al. 2011a, GRB Coordinates Network, 12083

Beardmore, A. P., Campana, S., Kennea, J. A., \& Swenson, C. A. 2011b, ATel, 3454

Bildsten, L. 1998, in NATO ASIC Proc. 515, The Many Faces of Neutron Stars., ed. R. Buccheri, J. van Paradijs, \& A. Alpar (Dordrecht: Kluwer), 419

Bochanski, J. J., Hawley, S. L., Covey, K. R., et al. 2010, AJ, 139, 2679

Boirin, L., Keek, L., Méndez, M., et al. 2007, A\&A, 465, 559

Brown, E. F., Bildsten, L., \& Rutledge, R. E. 1998, ApJ, 504, L95

Brown, E. F., \& Cumming, A. 2009, ApJ, 698, 1020

Burrows, D. N., Hill, J. E., Nousek, J. A., et al. 2005, Space Sci. Rev., 120, 165

Cackett, E. M., Brown, E. F., Cumming, A., et al. 2010a, ApJ, 722, L137

Cackett, E. M., Brown, E. F., Miller, J. M., \& Wijnands, R. 2010b, ApJ, 720,1325

Cackett, E. M., Wijnands, R., Miller, J. M., Brown, E. F., \& Degenaar, N. 2008, ApJ, 687, L87

Campana, S., Ferrari, N., Stella, L., \& Israel, G. L. 2005, A\&A, 434, L9

Chelovekov, I. V., Grebenev, S. A., \& Sunyaev, R. A. 2006, Astron. Lett., 32,456

Chen, W., Shrader, C. R., \& Livio, M. 1997, ApJ, 491, 312

Chenevez, J., Falanga, M., Kuuklers, E., et al. 2008, in Proc. 7th INTEGRAL Workshop (Copenhagen: SISSA), 33

Colpi, M., Geppert, U., Page, D., \& Possenti, A. 2001, ApJ, 548, L175

Cooper, R. L., \& Narayan, R. 2007, ApJ, 661, 468

Cornelisse, R., in 't Zand, J. J. M., Verbunt, F., et al. 2003, A\&A, 405, 1033

Degenaar, N., Brown, E. F., \& Wijnands, R. 2011a, MNRAS, 418, L152

Degenaar, N., Jonker, P. G., Torres, M. A. P., et al. 2010, MNRAS, 404, 1591

Degenaar, N., Patruno, A., \& Wijnands, R. 2012a, ApJ, 756, 148

Degenaar, N., \& Wijnands, R. 2009, A\&A, 495, 547

Degenaar, N., \& Wijnands, R. 2011, MNRAS, 414, L50

Degenaar, N., \& Wijnands, R. 2012, MNRAS, 422, 581

Degenaar, N., Wijnands, R., Cackett, E. M., et al. 2012b, A\&A, 545, 49

Degenaar, N., Wijnands, R., \& Kaur, R. 2011b, MNRAS, 414, L104

Degenaar, N., Wijnands, R., Wolff, M. T., et al. 2009, MNRAS, 396, L26

Degenaar, N., Wolff, M. T., Ray, P. S., et al. 2011c, MNRAS, 412, 1409

Degenaar, N., Yang, Y. J., \& Wijnands, R. 2011d, ATel, 3741

Díaz Trigo, M., Boirin, L., Costantini, E., Méndez, M., \& Parmar, A. 2011, A\&A, 528,150

Drilling, J. S., \& Landolt, A. U. 2000, in Normal Stars, ed. A. N. Cox (New York: Springer), 38

Evans, P. A., Beardmore, A. P., Page, K. L., et al. 2009, MNRAS, 397, 1177

Falanga, M., Belloni, T., \& Campana, S. 2006, A\&A, 456, L5

Falanga, M., Chenevez, J., Cumming, A., et al. 2008, A\&A, 484, 43

Falanga, M., Cumming, A., Bozzo, E., \& Chenevez, J. 2009, A\&A, 496, 333

Fridriksson, J. K., Homan, J., Wijnands, R., et al. 2010, ApJ, 714, 270

Fridriksson, J. K., Homan, J., Wijnands, R., et al. 2011, ApJ, 736, 162

Fujimoto, M. Y., Hanawa, T., \& Miyaji, S. 1981, ApJ, 247, 267

Fushiki, I., Taam, R. E., Woosley, S. E., \& Lamb, D. Q. 1992, ApJ, 390, 634

Galloway, D. K., Muno, M. P., Hartman, J. M., Psaltis, D., \& Chakrabarty, D. 2008, ApJS, 179, 360

Garcia, M. R., McClintock, J. E., Narayan, R., et al. 2001, ApJ, 553, L47

Gehrels, N. 1986, ApJ, 303, 336
Gorosabel, J., Terron, V., Ferrero, P., et al. 2011, GRB Coordinates Network, 12101

Groth, E. J. 1975, ApJS, 29, 285

Gupta, S., Brown, E. F., Schatz, H., Möller, P., \& Kratz, K.-L. 2007, ApJ, 662,1188

Güver, T., \& Özel, F. 2009, MNRAS, 400, 2050

Haensel, P., \& Zdunik, J. L. 1990, A\&A, 227, 431

Haensel, P., \& Zdunik, J. L. 2008, A\&A, 480, 459

Halpern, J. P., \& Skinner, J. 2011, ATel, 3742

Hawley, S. L., Allred, J. C., Johns-Krull, C. M., et al. 2003, ApJ, 597, 535

Heinke, C. O., Jonker, P. G., Wijnands, R., Deloye, C. J., \& Taam, R. E. 2009, ApJ, 691, 1035

Im, M., Kim, J. H., \& Choi, C. 2011, GRB Coordinates Network, 12090

in 't Zand, J. J. M., Bassa, C. G., Jonker, P. G., et al. 2008, A\&A, 485, 183

in 't Zand, J. J. M., Jonker, P. G., \& Markwardt, C. B. 2007, A\&A, 465, 953

in 't Zand, J. J. M., Keek, L., Cumming, A., et al. 2009, A\&A, 497, 469

Jonker, P. G., Galloway, D. K., McClintock, J. E., et al. 2004, MNRAS, 354,666

Keek, L., \& in 't Zand, J. J. M. 2008, in Proc. 7th INTEGRAL Workshop (Copenhagen: SISSA), 32

Kennea, J. A., Krimm, H. A., Yamaoka, K., Nakahira, S., \& Burrows, D. N. 2011, ATel, 3567

Krimm, H. A., Barthelmy, S. D., Baumgartner, W., et al. 2011, ATel, 3455

Kuulkers, E. 2004, Nucl. Phys. B, 132, 466

Kuulkers, E., den Hartog, P. R., in 't Zand, J. J. M., et al. 2003, A\&A, 399, 663

Kuulkers, E., in 't Zand, J. J. M., \& Lasota, J.-P. 2009, A\&A, 503, 889

Kuulkers, E., in 't Zand, J. J. M., Atteia, J.-L., et al. 2010, A\&A, 514, A65

Lasota, J.-P. 2001, New Astron. Rev., 45, 449

Leahy, D. A., Darbro, W., Elsner, R. F., et al. 1983, ApJ, 266, 160

Lin, D., Remillard, R. A., \& Homan, J. 2009, ApJ, 696, 1257

Linares, M. 2009, PhD thesis, Sterrenkundig Instituut Anton Pannekoek,Univ. Amsterdam

Linares, M., Connaughton, V., Jenke, P., et al. 2012, ApJ, in press (arXiv:1209.6053)

Linares, M., van der Klis, M., \& Wijnands, R. 2007, ApJ, 660, 595

Linares, M., Watts, A. L., Wijnands, R., et al. 2009, MNRAS, 392, L11

Lubiński, P. 2009, A\&A, 496, 557

Markwardt, C. B., Barthelmy, S. D., Baumgartner, W. H., et al. 2011, GRB Coordinates Network, 12086

Menou, K., Esin, A. A., Narayan, R., et al. 1999, ApJ, 520, 276

Nakahira, S., Yamaoka, K., Sugizaki, M., et al. 2011, ATel, 3548,

Nelson, L. A., Rappaport, S. A., \& Joss, P. C. 1986, ApJ, 304, 231

Page, D., Lattimer, J. M., Prakash, M., \& Steiner, A. W. 2004, ApJS, 155, 623

Page, D., \& Reddy, S. 2012, arXiv:1201.5602

Peng, F., Brown, E. F., \& Truran, J. W. 2007, ApJ, 654, 1022

Poole, T. S., Breeveld, A. A., Page, M. J., et al. 2008, MNRAS, 383, 627

Roming, P. W. A., Kennedy, T. E., Mason, K. O., et al. 2005, Space Sci. Rev., 120,95

Rutledge, R. E., Bildsten, L., Brown, E. F., et al. 2002, ApJ, 580, 413

Rutledge, R. E., Bildsten, L., Brown, E. F., Pavlov, G. G., \& Zavlin, V. E. 1999, ApJ, 514, 945

Schatz, H., Aprahamian, A., Barnard, V., et al. 2001, Phys. Rev. Lett, 86, 3471

Shternin, P. S., Yakovlev, D. G., Haensel, P., \& Potekhin, A. Y. 2007, MNRAS, 382, L43

Strohmayer, T., \& Bildsten, L. 2006, in New Views of Thermonuclear Bursts, ed. M. Lewin \& W. H. G. van der Klis (Cambridge: Cambridge University Press), 113

Suleimanov, V., Poutanen, J., \& Werner, K. 2011, A\&A, 527, A139

Trap, G., Falanga, M., Goldwurm, A., et al. 2009, A\&A, 504, 501

Tueller, J., Barthelmy, S., Burrows, D., et al. 2005a, ATel, 668

Tueller, J., Barthelmy, S., Burrows, D., et al. 2005b, ATel, 669

van der Klis, M. 1994, ApJS, 92, 511

Vaughan, B. A., van der Klis, M., Wood, K. S., et al. 1994, ApJ, 435, 362

Walter, R., Rohlfs, R., Meharga, M. T., et al. 2010, in Proc. 8th INTEGRAL Workshop (Dublin: SISSA), 162

Watts, A. L. 2012, ARA\&A, 50, 609

Wiersema, K., Levan, A., Tanvir, N., \& Tibbetts-Harlow, K. 2011, ATel, 3740

Wijnands, R. 2005, in Progress in Neutron Star Research, ed. A. P. Wass (New York: Nova Science Publishers)

Wijnands, R., Heinke, C. O., Pooley, D., et al. 2005a, ApJ, 618, 883

Wijnands, R., Homan, J., Heinke, C. O., Miller, J. M., \& Lewin, W. H. G. 2005b, ApJ, 619, 492

Wijnands, R., in 't Zand, J. J. M., Rupen, M., et al. 2006, A\&A, 449, 1117

Wijnands, R., Miller, J. M., Markwardt, C., Lewin, W. H. G., \& van der Klis, M. 2001, ApJ, 560, L159

Wijnands, R., Nowak, M., Miller, J. M., et al. 2003, ApJ, 594, 952

Yakovlev, D. G., \& Pethick, C. J. 2004, ARA\&A, 42, 169 